\title{
Coordinate-free characterization of homogeneous polynomials with isolated singularities
}

\author{
Irene Chen, Ke-Pao Lin, Stephen Yau and Huaiqing Zuo
}

\begin{abstract}
The Durfee conjecture, proposed in 1978, relates two important invariants of isolated hypersurface singularities by a famous inequality; however, the inequality in this conjecture is not sharp. In 1995, Yau announced his conjecture which proposed a sharp inequality. The Yau conjecture characterizes the conditions under which an affine hypersurface with an isolated singularity at the origin is a cone over a nonsingular projective hypersurface; in other words, the conjecture gives a coordinate-free characterization of when a convergent power series is a homogeneous polynomial after a biholomorphic change of variables. In this paper, we have proved that if $p_{g}>0$, then $5 ! p_{g} \leq \mu-p(v), p(v)=(v-1)^{5}-$ $v(v-1) \ldots(v-4)$ and $p_{g}, \mu$ and $v$ are, respectively, the geometric genus, the Milnor number, and the multiplicity of the isolated singularity at the origin of a weighted homogeneous polynomial. As a consequence, we prove that the Yau conjecture holds for $n=5$ if $p_{g}>0$. In the process, we have also defined yet another sharp upper bound for the number of positive integral points within a five-dimensional simplex.
\end{abstract}

\section{Introduction}

Let $\Delta_{n}$ be an $n$-dimensional real right-angled simplex defined by the inequality

$$
\frac{x_{1}}{a_{1}}+\frac{x_{2}}{a_{2}}+\cdots+\frac{x_{n}}{a_{n}} \leq 1
$$

where $x_{1} \geq 0, \ldots, x_{n} \geq 0$ and $a_{1} \geq a_{2} \geq \cdots \geq a_{n} \geq 1$. Define $P_{n}$ to be the number of positive integral points in $\Delta_{n}$, as shown below:

$$
P_{n}=\#\left\{\left(x_{1}, x_{2}, \ldots, x_{n}\right) \in \mathbb{Z}_{+}^{n} \mid \frac{x_{1}}{a_{1}}+\frac{x_{2}}{a_{2}}+\cdots+\frac{x_{n}}{a_{n}} \leq 1\right\}
$$


Define $Q_{n}$ to be the number of nonnegative integral points in $\Delta_{n}$, as shown below:

$$
Q_{n}=\#\left\{\left(x_{1}, x_{2}, \ldots, x_{n}\right) \in\left(\mathbb{Z}_{+} \cup\{0\}\right)^{n} \mid \frac{x_{1}}{a_{1}}+\frac{x_{2}}{a_{2}}+\cdots+\frac{x_{n}}{a_{n}} \leq 1\right\} .
$$

The problem of obtaining the numbers $P_{n}$ and $Q_{n}$ has occupied mathematicians for decades, simply because a sharp upper estimate of the former would benefit those in singularity theory and a sharp upper estimate of the latter would benefit those in number theory. Granville [3] has stated that an estimate of $Q_{n}$ would help with finding large gaps between primes, and research done by $\mathrm{Xu}$ and Yau $[19,21]$ on the Durfee conjecture has shown that an estimate of $P_{n}$ does aid mathematicians in singularity theory. These two different numbers are tied together through the equation

$$
P_{n}\left(a_{1}, a_{2}, \ldots, a_{n}\right)=Q_{n}\left(a_{1}(1-a), a_{2}(1-a), \ldots, a_{n}(1-a)\right)
$$

where $a=\frac{1}{a_{1}}+\cdots+\frac{1}{a_{n}}$. Thus, if one discovers a new estimate for $P_{n}$, a new estimate for $Q_{n}$ will also be present.

In 1899, Pick [15] discovered a formula for $Q_{2}$ :

$$
Q_{2}=\operatorname{area}(\triangle)+\frac{\left|\partial \triangle \cap \mathbb{Z}^{2}\right|}{2}+1
$$

where $\partial \triangle$ is the boundary of the simplex and $\left|\partial \triangle \cap \mathbb{Z}^{n}\right|$ is the number of integral points on the boundary. In 1951, Mordell [13] discovered the formula for $Q_{3}$ using Dedekind sums, but the real breakthrough occurred when Ehrhart [2] proved a polynomial of degree $n$ could calculate the number of nonnegative lattice points in $n$-dimensional simplex. However, his formula is only effective when the coefficients of every term are known. Starting in 1939, attempts were made to find lower and upper bounds for $Q_{n}$ instead of a precise formula. It was later discovered by Lehmer [5] that if $a=a_{1}=$ $a_{2}=\cdots=a_{n}$,

$$
Q_{n}=\left(\begin{array}{c}
{[a]+n} \\
n
\end{array}\right)
$$

From that formula, it is very natural to introduce the following definition for a sharp estimate $R_{n}$ of $Q_{n}$ [18]. The estimate is sharp if

$$
\left.R_{n}\right|_{a_{1}=\cdots=a_{n}=a \in \mathbb{Z}}=\left(\begin{array}{c}
a+n \\
n
\end{array}\right) .
$$


In other words, any upper or lower bound is only considered to be a sharp estimate if and only if the exact number of lattice points in $n$-dimensional simplex is attained when $a=a_{1}=a_{2}=\cdots=a_{n} \in \mathbb{Z}$.

Let $(V, 0)$ be an isolated hypersurface singularity defined by holomorphic function $f:\left(\mathbb{C}^{n+1}, 0\right) \rightarrow(\mathbb{C}, 0)$. Let $\pi: M \rightarrow V$ be a resolution of the singularity at 0 . Define the geometric genus of the singularity $(V, 0)$ to be $p_{g}=\operatorname{dim} H^{n-1}(M, \mathcal{O})$. Let $\omega$ be a holomorphic $n$ forms on $V-\{0\}$. $\omega$ is said to be $L^{2}$ integrable if $\int_{W-\{0\}} \omega \wedge \bar{\omega}<\infty$ for sufficiently small relatively compact neighborhood $W$ of 0 in $V$. Let $L^{2}\left(V-\{0\}, \Omega^{n}\right)$ be the set of all $L^{2}$ integral holomorphic $n$ forms $V-\{0\}$, which is a linear subspace of $\Gamma(V-$ $\left.\{0\}, \Omega^{n}\right)$. Then it was proved that $p_{g}=\operatorname{dim} \Gamma\left(V-\{0\}, \Omega^{n}\right) / L^{2}\left(V-\{0\}, \Omega^{n}\right)$ (see [23]). The Milnor number of the singularity $(V, 0)$ is defined as

$$
\mu=\operatorname{dim} \mathbb{C}\left\{z_{0}, z_{1}, \ldots, z_{n}\right\} /\left(f_{z_{0}}, f_{z_{1}}, \ldots, f_{z_{n}}\right) .
$$

The multiplicity of the singularity is defined to be the order of the lowest nonvanishing term in the power series Taylor expansion of $f$ at 0 . In singularity theory, Durfee [1] formed his famous conjecture. The conjecture states that for an isolated singularity $(V, 0)$ defined by a weighted homogeneous polynomial $f\left(z_{0}, z_{1}, z_{2}, \ldots, z_{n}\right)$,

$$
(n+1) ! p_{g} \leq \mu,
$$

where $p_{g}$ is the geometric genus of $V, \mu$ is the Milnor number, and equality holds if and only if $\mu=0$. A polynomial $f\left(z_{0}, z_{1}, z_{2}, \ldots, z_{n}\right)$ is a weighted homogeneous polynomial of the type $\left(w_{0}, w_{1}, w_{2}, \ldots, w_{n}\right)$, where $w_{0}, w_{1}$, $w_{2}, \ldots, w_{n}$ are fixed positive rational numbers, if $f$ can be expressed as a linear combination of monomials $z_{0}^{i_{0}} z_{1}^{i_{1}} \ldots z_{n}^{i_{n}}$ for which $\frac{i_{0}}{w_{0}}+\frac{i_{1}}{w_{1}}+\cdots+$ $\frac{i_{n}}{w_{n}}=1$. Furthermore, the Milnor number $\mu[12]$ can be expressed as $\left(w_{0}-1\right)$ $\left(w_{1}-1\right) \ldots\left(w_{n}-1\right)$. As a consequence of the theorem of Merle-Teissier [14], we know that in case of isolated singularity defined by a weighted homogeneous polynomial, computing the geometric genus is equivalent to counting the number of positive integral points in a tetrahedron.

The next sharp estimate to be constructed was the GLY conjecture, formulated by Lin et al. [9]. It has two different parts: the sharp estimate and the rough estimate. However, before we state it, it is convenient to introduce the Sterling numbers first:

$$
S_{k}^{n-1}=\sum_{1 \leq i_{1}<i_{2}<\cdots<i_{k} \leq n-1} i_{1} i_{2} \ldots i_{k}, S_{0}^{n-1}=1, S_{n-1}^{n-1}=1 \cdot 2 \ldots(n-1),
$$


where $i_{1}, i_{2}, \ldots, i_{k}$ are integers. It has the following property:

$$
\begin{aligned}
x(x-1)(x-2) \ldots(x-n+1) & \\
= & x^{n}-\left(\sum_{i_{1}=1}^{n-1} i_{1}\right) x^{n-1}+(-1)^{2}\left(\sum_{1 \leq i_{1}<i_{2} \leq n-1} i_{1} i_{2}\right) x^{n-2} \\
& +\cdots+(-1)^{k}\left(\sum_{i \leq i_{1}<i_{2}<\cdots<i_{k} \leq n-1} i_{1} i_{2} \ldots i_{k}\right) x^{n-k} \\
& +\cdots+(-1)^{n-1}\left(\prod_{i=1}^{n-1} i\right) x \\
= & x^{n}+(-1) S_{1}^{n-1} x^{n-1}+(-1)^{2} S_{2}^{n-1} x^{n-2} \\
& +\cdots+(-1)^{k} S_{k}^{n-1} x^{n-k}+\cdots+(-1)^{n-1} S_{n-1}^{n-1} x .
\end{aligned}
$$

Let $a_{1}, a_{2}, \ldots, a_{n}$ be positive real numbers. We shall denote

$$
\begin{aligned}
A_{n-k}^{n} & =\left(\prod_{i=1}^{n} a_{i}\right) \sum_{1 \leq i_{1}<i_{2}<\cdots<i_{k} \leq n} \frac{1}{a_{i_{1}} a_{i_{2}} \ldots a_{i_{k}}} \\
A_{1}^{n} & =\sum_{i=1}^{n} a_{i}, A_{0}^{n}=1 .
\end{aligned}
$$

Observe that $A_{n-k}^{n}$ is a polynomial in $a_{1}, a_{2}, \ldots, a_{n}$ of degree $n-k$.

Conjecture 1.1 (Granville-Lin-Yau (GLY) conjecture [9, 18]). Let $P_{n}=\#\left\{\left(x_{1}, x_{2}, \ldots, x_{n}\right) \in \mathbb{Z}_{+}^{n}: \frac{x_{1}}{a_{1}}+\frac{x_{2}}{a_{2}}+\cdots+\frac{x_{n}}{a_{n}} \leq 1\right\}$, where $a_{1} \geq a_{2}$ $\geq \cdots \geq a_{n} \geq 1$ are real numbers. If $n \geq 3$, then

(1) Rough (general) upper estimate: For all $a_{n} \geq 1$,

$$
n ! P_{n} \leq q_{n}:=\prod_{i=1}^{n}\left(a_{i}-1\right)
$$

where equality holds if and only if $a_{1}=a_{2}=\cdots=a_{n}=$ integer. 
(2) Sharp upper estimate: For $a_{n}$ sufficiently large, there exists an integer $\beta(n)$ that depends on $n$ such that when $a_{n} \geq \beta(n)$, then

$$
\begin{aligned}
n ! P_{n} \leq & A_{n}^{n}+(-1) \frac{S_{1}^{n-1}}{n} A_{n-1}^{n}+(-1)^{2} \frac{S_{2}^{n-1}}{\left(\begin{array}{c}
n-1 \\
1
\end{array}\right)} A_{n-2}^{n-1}+(-1)^{3} \frac{S_{3}^{n-1}}{\left(\begin{array}{c}
n-1 \\
2
\end{array}\right)} A_{n-3}^{n-1} \\
& +\cdots+(-1)^{k-1} \frac{S_{k+1}^{n-1}}{\left(\begin{array}{c}
n-1 \\
k
\end{array}\right)} A_{n-k-1}^{n-1}+\cdots+(-1)^{n-1} \frac{S_{n-1}^{n-1}}{\left(\begin{array}{c}
n-1 \\
n-2
\end{array}\right)} A_{1}^{n-1},
\end{aligned}
$$

and equality holds if and only if $a_{1}=a_{2}=\cdots=a_{n} \in \mathbb{Z}_{+}$.

The GLY conjecture was proven by $\mathrm{Xu}$ and Yau for $n=3$ [20] and $n=4$ [22], for $n=5$, see [4], [6] and [8], Wang and Yau for $3 \leq n \leq 6$ [18], where $\beta(n)=n-1$ for $3 \leq n \leq 6$. The rough GLY upper estimate for all $n$ was proven in [24].

In particular, the following theorems will be used frequently.

Theorem 1.1. Let $a \geq b \geq c \geq d \geq 2$, and $P_{4}$ be the number of positive integral solutions of $\frac{x}{a}+\frac{y}{b}+\frac{z}{c}+\frac{w}{d} \leq 1$, i.e., $P_{4}=\sharp\left\{(x, y, z, w) \in Z_{+}^{4}: \frac{x}{a}+\right.$ $\left.\frac{y}{b}+\frac{z}{c}+\frac{w}{d} \leq 1\right\}$. If $P_{4}>0$, then

$$
\begin{aligned}
24 P_{4} \leq f_{4}(a, b, c, d):= & a b c d-\frac{3}{2}(a b c+a b d+a c d+b c d) \\
& +\frac{11}{3}(a b+a c+b c)-2(a+b+c)
\end{aligned}
$$

and equality is attained if and only if $a=b=c=d=$ integer.

If $a \geq b \geq c \geq d \geq 3$ or $a \geq 8, b \geq 6, c \geq 4, d \geq 2$, then Theorem 1.1 is true without the condition $P_{4}>0$ and equality is attained if and only if $a=b=c=d=$ integer.

Theorem 1.2. Let $a \geq b \geq c \geq d \geq 1$, and $P_{4}$ be the number of positive integral solutions of $\frac{x}{a}+\frac{y}{b}+\frac{z}{c}+\frac{w}{d} \leq 1$, i.e., $P_{4}=\sharp\left\{(x, y, z, w) \in Z_{+}^{4}: \frac{x}{a}+\right.$ $\left.\frac{y}{b}+\frac{z}{c}+\frac{w}{d} \leq 1\right\}$. Define $\mu=(a-1)(b-1)(c-1)(d-1)$, then

$$
\begin{aligned}
24 P_{4} \leq \mu= & a b c d-(a b c+a b d+a c d+b c d) \\
& +(a b+a c+a d+b c+b d+c d)-(a+b+c+d)+1 .
\end{aligned}
$$


Theorem $1.3(\mathbf{L u}-\mathbf{Y a}-\mathbf{Z u}[\mathbf{1 1}])$. Let $a \geq b \geq c \geq d \geq 1$, and $P_{4}$ be the number of positive integral solutions of $\frac{x}{a}+\frac{y}{b}+\frac{z}{c}+\frac{w}{d} \leq 1$, i.e., $P_{4}=\sharp\{(x, y$, $\left.z, w) \in Z_{+}^{4}: \frac{x}{a}+\frac{y}{b}+\frac{z}{c}+\frac{w}{d} \leq 1\right\}$. Define $\mu=(a-1)(b-1)(c-1)(d-1)$, if $P_{4}>0$, then

$$
\begin{aligned}
24 P_{4} \leq g_{4}(a, b, c, d):= & (a-1)(b-1)(c-1)(d-1) \\
& -(d-1)^{4}+d(d-1)(d-2)(d-3)
\end{aligned}
$$

and equality is attained if and only if $a=b=c=d=$ integer.

The GLY conjecture was the first major step towards proving the following conjecture made by Yau in 1995 [24]:

Conjecture 1.2. Let $f:\left(\mathbb{C}^{n}, 0\right) \rightarrow(\mathbb{C}, 0)$ be a weighted homogeneous polynomial with an isolated singularity at the origin. Let $\mu, p_{g}$ and $v$ be the Milnor number, geometric genus and multiplicity of the singularity $V=$ $\{z: f(z)=0\}$, then

$$
\mu-p(v) \geq n ! p_{g}
$$

where $p(v)=(v-1)^{n}-v(v-1) \ldots(v-n+1)$, and equality holds if and only if $f$ is a homogeneous polynomial.

This conjecture is a sharp estimate that holds without the restriction of the sharp GLY estimate, $a_{n} \geq \beta(n)$, and it also has some important applications in geometry.

The Yau conjecture was already proven for the cases $n=3$ [20] and $n=4[10]$. In this paper, we aim to prove the Yau conjecture for $n=5$, which is stated below.

Theorem 1.4. Let $f:\left(\mathbb{C}^{5}, 0\right) \rightarrow(\mathbb{C}, 0)$ be a weighted homogeneous polynomial with an isolated singularity at the origin. Let $\mu, p_{g}$ and $v$ be the Milnor number, geometric genus and multiplicty of the singularity $V=\{z: f(z)=$ $0\}$. If $p_{g}>0$ then

$$
\mu-p(v) \geq 5 ! p_{g}
$$

where $p(v)=(v-1)^{5}-v(v-1) \ldots(v-4)$, and equality holds if and only if $f$ is a homogeneous polynomial.

As a corollary of Theorem 1.4, we have the following coordinate-free characterization of homogeneous polynomials with isolated singularities. 
Corollary. Let $f:\left(\mathbb{C}^{5}, 0\right) \rightarrow(\mathbb{C}, 0)$ be a holomorphic function with an isolated singularity at the origin. Let $\mu, p_{g}$ and $v$ be the Milnor number, the geometric genus and multiplicity of the singularity $V=\{z: f(z)=0\}$ at the origin. Let $\tau$ be the dimension of the semi-universal deformation space of $(V, 0)$ which is $\operatorname{dim} \mathbb{C}\left\{z_{1}, \ldots, z_{5}\right\} /\left(f, f_{z_{1}}, \ldots, f_{z_{5}}\right)$. Assume that $p_{g}>0$, then, after a biholomorphic change of coordinates, $f$ is a homogeneous polynomial if and only if $\mu-(v-1)^{5}+v(v-1)(v-2)(v-3)(v-4)=5 ! P_{g}$ and $\mu=\tau$.

It was observed by Saeki [16] (also see [20]) that the multiplicity $v$ is $\inf \left\{n \in \mathbb{Z}_{+}: n\right\} \geq \inf \left\{w_{0}, w_{1}, w_{2}, w_{3}, w_{4}\right\}$, where $w_{i}$ is a weight of $x_{i}$ in $f$. Therefore, proving our Main Theorem, Theorem 1.4 above, is akin to proving the following theorem about the number of integral points within a fivedimensional simplex, in view of the Theorem 2.5 in Section 2.5 of this paper.

Theorem 1.5. Let $a \geq b \geq c \geq d \geq e>1$ be real numbers and let $P_{5}$ be the number of positive integral solutions of $\frac{x}{a}+\frac{y}{b}+\frac{z}{c}+\frac{v}{d}+\frac{w}{e} \leq 1$, i.e., $P_{5}=$ $\#\left\{(x, y, z, v, w) \in \mathbb{Z}_{+}^{5}: \frac{x}{a}+\frac{y}{b}+\frac{z}{c}+\frac{v}{d}+\frac{w}{e} \leq 1\right\}$. Define $\mu=(a-1)(b-1)(c$ $-1)(d-1)(e-1)$. If $P_{5}>0$, then

$$
5 ! P_{5} \leq \mu-\left(5 v^{4}-25 v^{3}+40 v^{2}-19 v-1\right),
$$

where $v$ is the multiplicity calculated by $v=e$, if $e$ is an integer, or by $v=$ $[e]+1=e-\beta+1$, if $e$ is not an integer and $\beta$ is either $\frac{e}{a}, \frac{e}{b}, \frac{e}{c}$ or $\frac{e}{d}$, where $[e]=e-\beta$ is the integral part of $e$. Equality holds if and only if $a=b=c=$ $d=e$ are all integers.

Although the idea of the proof of our theorem is very simple, our proof is quite delicate. We try to estimate $P_{4}$ on hyperplanes parallel to the $x y z v$ plane by using the upper bounds in the four-dimensional case provided by Theorems 1.1 to 1.3 above and sum these estimates up to get $P_{5}$. In order to avoid the negative amount difficulty in $f_{4}(a, b, c, d)$ and $g_{4}(a, b, c, d)$ which may happen when $d<3$, we need a careful analysis of the last two or three hyperplanes. For this reason, Theorems 1.1 to 1.3 are needed to deal with the problem. Our main theorem follows from a careful analysis of this sum. All the computations in this paper are done by Maple V.

By level $w=k$, we shall mean the intersection of the tetrahedron in Theorem 1.5 with the hyperplane $w=k$. In our tetrahedron, $w=k$ points are in following four-dimensional tetrahedron

$$
\frac{x}{a\left(1-\frac{k}{e}\right)}+\frac{y}{b\left(1-\frac{k}{e}\right)}+\frac{z}{c\left(1-\frac{k}{e}\right)}+\frac{v}{d\left(1-\frac{k}{e}\right)} \leq 1 .
$$


We can use Theorem 1.1 to bound the number of positive integral solutions at level $w=k$ if $d\left(1-\frac{k}{e}\right) \geq 3$ or $d\left(1-\frac{k}{e}\right) \geq 2$ with the condition there exist positive integral solutions at this level. We can use Theorem 1.2 to bound the number of positive integral solutions at level $w=k$ if $d\left(1-\frac{k}{e}\right)>1$ without the condition there exist positive integral solutions at this level. We can use Theorem 1.3 to bound the number of positive integral solutions at level $w=$ $k$ if $d\left(1-\frac{k}{e}\right)>1$ with the condition there exist positive integral solutions at this level. In order to do this, we will split up the proof into five main cases, depending on the value of $e$, and utilize Theorems 1.1 to 1.3 carefully in each case:

(I) $e \geq 5, e \in \mathbb{Z}_{+}$,

(II) $e>4, e \notin \mathbb{Z}_{+}$,

(III) $4 \geq e>3$

(IV) $3 \geq e>2$,

(V) $2 \geq e>1$.

We slice the five-dimensional simplex into the hyperplanes, $w=1, w=2, \ldots$, $w=e-\beta-1$ and $w=e-\beta$. It is obvious there are no positive integral solutions at level $w=e-\beta$. At level $w=e-\beta-2$, the defining inequality of the simplex becomes $\frac{x}{\frac{a}{e}(\beta+2)}+\frac{y}{\frac{b}{e}(\beta+2)}+\frac{z}{\frac{c}{e}(\beta+2)}+\frac{v}{\frac{d}{e}(\beta+2)} \leq 1$. If $\frac{d}{e}(\beta+$ $2)>3$, then Theorem 1.1 can be used. If $\frac{d}{e}(\beta+2)<3$ with the condition there are positive integral solutions at this level, then Theorem 1.3 can be used. If $\frac{d}{e}(\beta+2)<3$ with the condition there are no positive integral solutions at this level, then Theorem 1.2 can be used. At level $w=e-\beta-1$, the defining inequality of the simplex becomes $\frac{x}{\frac{a}{e}(\beta+1)}+\frac{y}{\frac{b}{e}(\beta+1)}+\frac{z}{\frac{c}{e}(\beta+1)}+$ $\frac{v}{\frac{d}{e}(\beta+1)} \leq 1$. In Case (II), we divide our proof into two cases depending on whether the tetrahedron

$$
\frac{x}{\frac{a}{e}(\beta+1)}+\frac{y}{\frac{b}{e}(\beta+1)}+\frac{z}{\frac{c}{e}(\beta+1)}+\frac{v}{\frac{d}{e}(\beta+1)} \leq 1
$$

at level $w=e-\beta-1$ has positive integral solutions or not.

Case (A). $\frac{a}{e}(1+\beta)<4$ or $\frac{b}{e}(1+\beta) \leq 3$ or $\frac{c}{e}(1+\beta) \leq 2$ or $\frac{d}{e}(1+\beta) \leq 1$.

In this case, there are no positive integral solutions on level $w=$ $e-\beta-1$.

Case (B). $\frac{a}{e}(1+\beta) \geq 4, \quad \frac{b}{e}(1+\beta)>3, \quad \frac{c}{e}(1+\beta)>2$ and $\frac{d}{e}(1+\beta)>1$, where $0<\beta<1$. 
In this case, there may exist positive integral solutions on level $w=$ $e-\beta-1$.

\section{Proof of the main Theorem 4}

\subsection{Case I}

We will first analyze the case that occurs when $e \geq 5$ and $e$ is an integer.

Proposition 2.1. Let $a \geq b \geq c \geq d \geq e \geq 5$ be real numbers. Consider $\frac{x}{a}+\frac{y}{b}+\frac{z}{c}+\frac{v}{d}+\frac{w}{e} \leq 1$. Let $P_{5}$ be the number of positive integral solutions of the above inequality, i.e., $P_{5}=\#\left\{(x, y, z, v, w) \in \mathbb{Z}_{+}^{5}: \frac{x}{a}+\frac{y}{b}+\frac{z}{c}+\frac{v}{d}+\right.$ $\left.\frac{w}{e} \leq 1\right\}$. Then,

$$
\begin{aligned}
120 P_{5} \leq & a b c d e-2(a b c d+a b c e+a b d e+a c d e+b c d e) \\
& +\frac{35}{4}(a b c+a b d+a c d+b c d)-\frac{25}{3}(a b+a c+a d+b c+b d+c d) \\
& +6(a+b+c+d) .
\end{aligned}
$$

The inequality above has been taken from the main theorem of [7].

Before we go any further, we should note the interesting properties of $P_{4}$ that the lemma below points out.

Lemma 2.1. Let $a \geq b \geq c \geq d \geq 1$ be real numbers and let $P_{4}=\#\{(x, y$, $\left.z, v) \in \mathbb{Z}_{+}^{4}: \frac{x}{a}+\frac{y}{b}+\frac{z}{c}+\frac{v}{d} \leq 1\right\}$. Then, the following statements hold:

(1) if $b \leq 3$, then $P_{4}=0$,

(2) if $c \leq 2$, then $P_{4}=0$.

The theorem below is basically the Yau conjecture for $n=5$ with the property that $e$ is an integer.

Theorem 2.1. Let $a \geq b \geq c \geq d \geq e \geq 4$ be real numbers with $e$ an integer. Consider $\frac{x}{a}+\frac{y}{b}+\frac{z}{c}+\frac{v}{d}+\frac{w}{e} \leq 1$. Let $P_{5}$ be the number of positive integral solutions of $\frac{x}{a}+\frac{y}{b}+\frac{z}{c}+\frac{v}{d}+\frac{w}{e} \leq 1$, i.e., $P_{5}=\#\left\{(x, y, z, v, w) \in \mathbb{Z}_{+}^{5}: \frac{x}{a}+\right.$ $\left.\frac{y}{b}+\frac{z}{c}+\frac{v}{d}+\frac{w}{e} \leq 1\right\}$. Define $\mu=(a-1)(b-1)(c-1)(d-1)(e-1)$. Then,

$$
\begin{aligned}
120 P_{5} & \leq \mu-\left.\left(5 v^{4}-25 v^{3}+40 v^{2}-19 v-1\right)\right|_{v=e} \\
& =(a-1)(b-1)(c-1)(d-1)(e-1)-\left(5 e^{4}-25 e^{3}+40 e^{2}-19 e-1\right)
\end{aligned}
$$

with equality if and only if $a=b=c=d=e=$ integer. 
Proof. Because Proposition 2.1 is a previously proven upper estimate for $P_{5}$, we only need to show that the right-hand side (RHS) of Theorem 2.1 is larger than the RHS of Proposition 2.1. Let $\Delta_{1}$ be the difference by subtracting the RHS of Proposition 2.1 from the RHS of Theorem 2.1. We only need to show $\Delta_{1} \geq 0$.

$$
\begin{aligned}
\Delta_{1}= & a b c d+a b c e+a b d e+a c d e+b c d e-\frac{31}{4}(a b c+a b d+a c d+b c d) \\
& +a b e+a c e+b c e+a d e+b d e+c d e+\frac{22}{3}(a b+a c+b c \\
& +a d+b d+c d)-a e-b e-c e-d e-5(a+b+c+d) \\
& -5 e^{4}+25 e^{3}-40 e^{2}+20 e .
\end{aligned}
$$

Let $A=\frac{a}{e}, B=\frac{b}{e}, C=\frac{c}{e}$ and $D=\frac{d}{e}$; we can now rewrite $\Delta_{1}$.

$$
\begin{aligned}
\Delta_{1}= & (A B C D+A B C+A B D+A C D+B C D-5) e^{4} \\
& -\frac{31}{4} e^{3}(A B C+A B D+A C D+B C D) \\
& +e^{3}(A B+A C+B C+A D+B D+C D+25) \\
& +\frac{22}{3} e^{2}(A B+A C+B C+A D+B D+C D) \\
& -e^{2}(A+B+C+D+40)-5 e(A+B+C+D-4) .
\end{aligned}
$$

Note that $\Delta_{1}$ is symmetric with respect to $A, B, C$ and $D$. We can then apply a method that we call the "partial differentiation test": we calculate the partial derivative with respect to all the variables first and then partial differentiate with respect to one less variable for each consecutive step until we have the expressions for the first-order partials, which are $\frac{\partial}{\partial A} \Delta_{1}, \frac{\partial}{\partial B} \Delta_{1}$, $\frac{\partial}{\partial C} \Delta_{1}$ and $\frac{\partial}{\partial D} \Delta_{1}$ in this case. As long as we show that those first-order partials are positive throughout the domain and that the function is positive at the minimum, we can conclude that the function, and therefore the difference, is positive throughout the domain.

First,

$$
\frac{\partial^{4} \Delta_{1}}{\partial A \partial B \partial C \partial D}=e^{4}>0
$$

for all $e>1$. Then, it follows that $\frac{\partial^{3} \Delta_{1}}{\partial A \partial B \partial C}$ is an increasing function of $D$ for $e>1, D \geq 1$, and at the minimum $D=1$,

$$
\left.\frac{\partial^{3} \Delta_{1}}{\partial A \partial B \partial C}\right|_{D=1}=e^{3}\left(2 e-\frac{31}{4}\right)>0,
$$


for $e>\frac{31}{8}$, so $\frac{\partial^{3} \Delta_{1}}{\partial A \partial B \partial C}$ must be positive throughout the domain $D \geq 1, e>$ $\frac{31}{8}$. Because $\frac{\partial^{2} \Delta_{1}}{\partial A \partial B}$ is symmetric with respect to $C$ and $D, \frac{\partial^{3} \Delta_{1}}{\partial A \partial B \partial D}$ must also be positive on the domain $C \geq 1, e>\frac{31}{8}$. Then, from knowing that both $\frac{\partial^{3} \Delta_{1}}{\partial A \partial B \partial C}$ and $\frac{\partial^{3} \Delta_{1}}{\partial A \partial B \partial D}$ are positive, $\frac{\partial^{2} \Delta_{1}}{\partial A \partial B}$ is increasing with respect to $C$ and $D$ for $C \geq D \geq 1$ and $e>\frac{31}{8}$. At the minimum $C=D=1$,

$$
\left.\frac{\partial^{2} \Delta_{1}}{\partial A \partial B}\right|_{C=D=1}=e^{2}\left(3 e^{2}-\frac{29}{2} e+\frac{22}{3}\right)>0,
$$

for $e>\frac{29+\sqrt{489}}{12}$ or $e \geq 5$, so $\frac{\partial^{2} \Delta_{1}}{\partial A \partial B}>0$ for $C \geq 1, D \geq 1$ and $e \geq 5$. Because $\frac{\partial \Delta_{1}}{\partial A}$ is symmetric with respect to $B, C$ and $D$, both $\frac{\partial^{2} \Delta_{1}}{\partial A \partial C}$ and $\frac{\partial^{2} \Delta_{1}}{\partial A \partial D}$ must also be positive on the respective domains of $B \geq 1, D \geq 1, e \geq 5$ and $B \geq 1$, $C \geq 1, e \geq 5$ and $\frac{\partial \Delta_{1}}{\partial A}$ is an increasing function of $B, C$ and $D$ for $B \geq 1$, $C \geq 1, D \geq 1$ and $e \geq 5$. At the minimum of $B=C=D=1$,

$$
\left.\frac{\partial \Delta_{1}}{\partial A}\right|_{B=C=D=1}=e\left(4 e^{3}-\frac{81}{4} e^{2}+21 e-5\right)>0,
$$

for $e \geq 5$. Then, we know that $\Delta_{1}$ is increasing with respect to $A$ for $B \geq 1$, $C \geq 1, D \geq 1$ and $e \geq 5$. Because $\Delta_{1}$ is symmetric with respect to $A, B$, $C$ and $D, \Delta_{1}$ must also be increasing with respect to $B, C$ and $D$ for the respective domains of $A \geq 1, C \geq 1, D \geq 1, e \geq 5 ; A \geq 1, B \geq 1, D \geq 1$, $e \geq 5$; and $A \geq 1, B \geq 1, C \geq 1, e \geq 5$. Evaluated at the minimum of $A=$ $B=C=D=1$,

$$
\left.\Delta_{1}\right|_{A=B=C=D=1}=0,
$$

hence $\Delta_{1}$ is nonnegative on the domain $A \geq 1, B \geq 1, C \geq 1, D \geq 1$ and $e \geq 5$, and therefore the RHS of Theorem 2.1 is greater than or equal to the RHS of Proposition 2.1.

\subsection{Case V}

Theorem 2.2. Let $a \geq b \geq c \geq d \geq e$ and $e \in(1,2]$ be real numbers. Consider $\frac{x}{a}+\frac{y}{b}+\frac{z}{c}+\frac{v}{d}+\frac{w}{e} \leq 1$. Let $P_{5}$ be the number of positive integral solutions of $\frac{x}{a}+\frac{y}{b}+\frac{z}{c}+\frac{v}{d}+\frac{w}{e} \leq 1$, i.e., $P_{5}=\#\left\{(x, y, z, v, w) \in \mathbb{Z}_{+}^{5}: \frac{x}{a}+\frac{y}{b}+\right.$ $\left.\frac{z}{c}+\frac{v}{d}+\frac{w}{e} \leq 1\right\}$. Define $\mu=(a-1)(b-1)(c-1)(d-1)(e-1)$. Then, if $P_{5}>0$,

$$
\begin{aligned}
120 P_{5} & \leq \mu-\left.\left(5 v^{4}-25 v^{3}+40 v^{2}-19 v-1\right)\right|_{v=2} \\
& =(a-1)(b-1)(c-1)(d-1)(e-1)-1
\end{aligned}
$$

with equality if and only if $a=b=c=d=e=$ integer. 
Proof. In this case, $e \in(1,2]$ and there are two levels to consider, $k=1$ and $k=2$. When $k=2$, the inequality $\frac{x}{a}+\frac{y}{b}+\frac{z}{c}+\frac{v}{d}+\frac{k}{e} \leq 1$ has no positive solutions, and when $k=1$, the set of positive solutions to the inequality contains the point $(1,1,1,1,1)$ if we assume $P_{5}>0$. If $\frac{1}{a}+\frac{1}{b}+\frac{1}{c}+\frac{1}{d} \leq 1-$ $\frac{1}{e} \triangleq \alpha$, then $\alpha \in\left(0, \frac{1}{2}\right]$ since $e \in(1,2]$. Let $A=a \alpha, B=b \alpha, C=c \alpha$, and $D=d \alpha$ and the following restrictions hold:

$$
A \geq 4, B \geq 3, C \geq 2, D>1
$$

since $\frac{1}{D}<1, \frac{2}{C} \leq \frac{1}{C}+\frac{1}{D} \leq 1, \frac{3}{B} \leq \frac{1}{B}+\frac{1}{C}+\frac{1}{D} \leq 1$ and $\frac{4}{A} \leq \frac{1}{A}+\frac{1}{B}+\frac{1}{C}+$ $\frac{1}{D} \leq 1$. Then, we have

$$
\begin{aligned}
5 ! P_{4}(k=1) \leq & 5\left[(A-1)(B-1)(C-1)(D-1)-(D-1)^{4}\right. \\
& +D(D-1)(D-2)(D-3)] .
\end{aligned}
$$

If we let $\Delta_{2}$ be the difference by subtracting the RHS of Theorem 2.2 from the RHS of the above inequality; then substituting $a=\frac{A}{\alpha}, b=\frac{B}{\alpha}, c=\frac{C}{\alpha}$, $d=\frac{D}{\alpha}, e=\frac{1}{1-\alpha}, \nu=2$, into $\Delta_{2}$, then all we have to do is apply the partial differentiation test for the expression

$$
\begin{aligned}
\Delta_{2}= & \frac{1}{\alpha^{3}(1-\alpha)}(A B C D-\alpha(A B C+A B D+A C D+B C D) \\
& +\alpha^{2}(A B+A C+B C+A D+B D+C D) \\
& -\alpha^{3}(1-4 A-4 B-4 C+5 A B+5 A C+5 B C \\
& -5 A B C-14 D+5 A D+5 B D-5 A B D+5 C D \\
& \left.-5 A C D-5 B C D+5 A B C D+25 D^{2}-10 D^{3}\right) \\
& +\alpha^{4}(2-5 A-5 B+5 A B-5 C+5 A C+5 B C \\
& -5 A B C-15 D+5 A D+5 B D-5 A B D+5 C D \\
& \left.\left.-5 A C D-5 B C D+5 A B C D+25 D^{2}-10 D^{3}\right)\right) \\
= & \frac{1}{\alpha^{3}(1-\alpha)} \Delta_{3}
\end{aligned}
$$

on the domain $A \geq 4, B \geq 3, C \geq 2, D>1$, and $\alpha \in\left(0, \frac{1}{2}\right]$. We see that

$$
\frac{\partial^{4} \Delta_{3}}{\partial A \partial B \partial C \partial D}=1-5 \alpha^{3}+5 \alpha^{4}>0
$$


for all $\alpha \in(0,1)$. Then, $\frac{\partial^{3} \Delta_{3}}{\partial A \partial B \partial C}$ is an increasing function of $D$ on $D \geq 1$, $\alpha \in(0,1)$ with a minimum at $D=1$.

$$
\left.\frac{\partial^{3} \Delta_{3}}{\partial A \partial B \partial C}\right|_{D=1}=1-\alpha>0
$$

for all $\alpha \in(0,1)$. It follows that $\frac{\partial^{3} \Delta_{3}}{\partial A \partial B \partial C}>0$ for all $D \geq 1, \alpha \in(0,1)$, and because $\frac{\partial^{2} \Delta_{3}}{\partial A \partial B}$ is symmetric with respect to $C$ and $D$, we also know $\frac{\partial^{3} \Delta_{3}}{\partial A \partial B \partial D}>$ 0 for all $C \geq 1, \alpha \in(0,1)$. Therefore, $\frac{\partial^{2} \Delta_{3}}{\partial A \partial B}$ is an increasing function with respect to $C, D$ for all $C \geq 1, D \geq 1, \alpha \in(0,1)$, and its minimum occurs at $C=D=1$.

$$
\left.\frac{\partial^{2} \Delta_{3}}{\partial A \partial B}\right|_{C=D=1}=1-2 \alpha+\alpha^{2}=(1-\alpha)^{2}>0,
$$

for all $\alpha \in(0,1)$. It follows that $\frac{\partial^{2} \Delta_{3}}{\partial A \partial B}>0$ for all $C \geq 1, D \geq 1, \alpha \in(0,1)$, and because $\frac{\partial \Delta_{3}}{\partial A}$ is symmetric with respect to $B, C$ and $D$, we also know $\frac{\partial^{2} \Delta_{3}}{\partial A \partial C}>0$ for all $B \geq 1, D \geq 1, \alpha \in(0,1)$ and $\frac{\partial^{2} \Delta_{3}}{\partial A \partial D}>0$ for all $B \geq 1, C \geq 1$, $\alpha \in(0,1)$. Therefore, $\frac{\partial \Delta_{3}}{\partial A}$ is an increasing function with respect to $B, C, D$ for all $B \geq 1, C \geq 1, D \geq 1, \alpha \in(0,1)$ with a minimum at $B=C=D=1$.

$$
\left.\frac{\partial \Delta_{3}}{\partial A}\right|_{B=C=D=1}=1-3 \alpha+3 \alpha^{2}+3 \alpha^{3}>(1-\alpha)^{3}>0
$$

for all $\alpha \in(0,1)$, so $\frac{\partial \Delta_{3}}{\partial A}>0$ for all $B \geq 1, \geq 1, \geq 1, \alpha \in(0,1)$. By the property that $\Delta_{3}$ is symmetric with respect to $A, B$ and $C$, we also have $\frac{\partial \Delta_{3}}{\partial B}>0$ for all $A \geq 1, C \geq 1, D \geq 1, \alpha \in(0,1)$ and $\frac{\partial \Delta_{3}}{\partial C}>0$ for all $A \geq 1, B \geq 1$, $D \geq 1, \alpha \in(0,1)$, and therefore, $\Delta_{3}$ is an increasing function with respect to $A, B$ and $C$. Meanwhile,

$$
\frac{\partial^{3} \Delta_{3}}{\partial D^{3}}=60 \alpha^{3}(1-\alpha)>0
$$

for all $\alpha \in(0,1)$, so $\frac{\partial^{2} \Delta_{3}}{\partial D^{2}}$ is an increasing function of $D$ for all $D \geq 1, \alpha \in$ $(0,1)$ with a minimum at $D=1$.

$$
\left.\frac{\partial^{2} \Delta_{3}}{\partial D^{2}}\right|_{D=1}=60 \alpha^{3}(1-\alpha)>0
$$

for all $\alpha \in(0,1)$, so $\frac{\partial^{2} \Delta_{3}}{\partial D^{2}}>0$ on the domain of $D \geq 1, \alpha \in(0,1)$, and therefore, $\frac{\partial \Delta_{3}}{\partial D}$ is an increasing function of $D$ for all $D \geq 1, \alpha \in(0,1)$. Furthermore, $\frac{\partial \Delta_{3}}{\partial D}$ is an increasing function with respect to $A, B, C$ and $D$ for 
$A \geq B \geq C \geq D \geq 1, \alpha \in(0,1)$, because $\frac{\partial \Delta_{3}}{\partial D}$ is symmetric with respect to $A, B$ and $C$. Then, the minimum of $\frac{\partial \Delta_{3}}{\partial D}$ occurs at $A=4, B=3, C=2$, $D=1$.

$$
\left.\frac{\partial \Delta_{3}}{\partial D}\right|_{A=4, B=3, C=2, D=1}=24-26 \alpha+9 \alpha^{2}-41 \alpha^{3}+40 \alpha^{4}>0
$$

for all $\alpha \in(0,1)$, and therefore, $\frac{\partial \Delta_{3}}{\partial D}>0$ for $A \geq 4, B \geq 3, C \geq 2, D \geq 1$, $\alpha \in(0,1)$, and $\Delta_{3}$ is an increasing function of $A, B, C, D$ for $A \geq 4, B \geq 3$, $C \geq 2, D \geq 1, \alpha \in(0,1)$. Thus, the minimum of $\Delta_{3}$ occurs at $A=4, B=3$, $C=2, D=1$ :

$$
\left.\Delta_{3}\right|_{A=4, B=3, C=2, D=1}=24-50 \alpha+35 \alpha^{2}-11 \alpha^{3}+2 \alpha^{4}>0
$$

for all $\alpha \in(0,1)$. Then, it follows that $\Delta_{3}>0$ for $A \geq 4, B \geq 3, C \geq 2$, $D \geq 1$ and $\alpha \in\left(0, \frac{1}{2}\right]$.

\subsection{Case IV}

Theorem 2.3. Let $a \geq b \geq c \geq d \geq e$ and $e \in(2,3]$ be real numbers. Consider $\frac{x}{a}+\frac{y}{b}+\frac{z}{c}+\frac{v}{d}+\frac{w}{e} \leq 1$. Let $P_{5}$ be the number of positive integral solutions of $\frac{x}{a}+\frac{y}{b}+\frac{z}{c}+\frac{v}{d}+\frac{w}{e} \leq 1$, i.e., $P_{5}=\#\left\{(v, w, x, y, z) \in \mathbb{Z}_{+}^{5}: \frac{x}{a}+\frac{y}{b}+\right.$ $\left.\frac{z}{c}+\frac{v}{d}+\frac{w}{e} \leq 1\right\}$. Define $\mu=(a-1)(b-1)(c-1)(d-1)(e-1)$. Then, if $P_{5}>0$,

$$
\begin{aligned}
120 P_{5} & \leq \mu-\left.\left(5 v^{4}-25 v^{3}+40 v^{2}-19 v-1\right)\right|_{v=3} \\
& =(a-1)(b-1)(c-1)(d-1)(e-1)-32
\end{aligned}
$$

with equality if and only if $a=b=c=d=e=$ integer.

Proof. In this case, $e \in(2,3]$, and there are two levels to consider: $k=1$, and $k=2$. Because of the criterion $P_{5}>0$, we know the level $k=1$ contains positive integral solutions, but due to the fact that we cannot draw any conclusions about the level $k=2$, we have two different subcases to consider:

(a) $P_{4}(2)=0$

(b) $P_{4}(2)>0$.

For subcase (a), the proof is almost exactly the same as the proof presented in Case V. Because $P_{5}>0$, the set of positive integral solutions includes the point $(1,1,1,1,1)$, and let $\frac{1}{a}+\frac{1}{b}+\frac{1}{c}+\frac{1}{d} \leq 1-\frac{1}{e} \triangleq \alpha, \alpha \in$ 
$\left(\frac{1}{2}, \frac{2}{3}\right]$, since $e \in(2,3]$. Let $A=a \alpha, B=b \alpha, C=c \alpha$ and $D=d \alpha$; then, the following restrictions apply:

$$
A \geq 4, B \geq 3, C \geq 2, D \geq \frac{\alpha}{1-\alpha}
$$

because $D=d \alpha \geq e \alpha=\frac{\alpha}{1-\alpha}$. With $\alpha \in\left(\frac{1}{2}, \frac{2}{3}\right], \frac{\alpha}{1-\alpha} \in(1,2]$, so we only need to show that the difference by subtracting the estimate provided in Case $\mathrm{V}$ by [11] from the RHS of Theorem 2.3, which is equal to $\frac{\Delta_{3}-31 \alpha^{3}(1-\alpha)}{\alpha^{3}(1-\alpha)}$ is positive for $A \geq 4, B \geq 3, C \geq 2, D \geq \frac{\alpha}{1-\alpha}$ and $\alpha \in\left(\frac{1}{2}, \frac{2}{3}\right]$. Notice that in Case V, we showed that all the first-order partial derivatives are positive for $A \geq 4$, $B \geq 3, C \geq 2, D \geq 1$ and $\alpha \in(0,1)$; the only difference was the minimum of $\Delta_{3}$. Note that the constant terms in Theorems 2.2 and 2.3 differ, so we should subtract $31 \alpha^{3}(1-\alpha)$ from $\Delta_{3}$ to make sure we are still proving Theorem 2.3 instead of Theorem 2.2. Then, we can substitute our new restrictions into the calculation of the minimum value of $\Delta_{3}-31 \alpha^{3}(1-\alpha)$ :

$$
\begin{aligned}
\Delta_{3} & -\left.31 \alpha^{3}(1-\alpha)\right|_{A=4, B=3, C=2, D=\frac{\alpha}{1-\alpha}} \\
& =\frac{\alpha^{2}\left(24-47 \alpha+6 \alpha^{2}+14 \alpha^{3}+13 \alpha^{4}\right)}{(\alpha-1)^{2}}>0
\end{aligned}
$$

for $\alpha \in\left(\frac{1}{2}, \frac{2}{3}\right]$. Then, it follows that $\Delta_{3}-31 \alpha^{3}(1-\alpha)>0$ for $A \geq 4, B \geq 3$, $C \geq 2, D \geq \frac{\alpha}{1-\alpha}$ and $\alpha \in\left(\frac{1}{2}, \frac{2}{3}\right]$.

For subcase (b), $P_{4}(2)>0$, which implies that $(1,1,1,1,2)$ is the smallest positive integer solution to the level $k=2$. Then, we have $\frac{1}{a}+\frac{1}{b}+\frac{1}{c}+\frac{1}{d} \leq$ $1-\frac{2}{e} \triangleq \alpha_{1}, \alpha_{1} \in\left(0, \frac{1}{3}\right]$, since $e \in(2,3]$. Let $A=a \alpha_{1}, B=b \alpha_{1}, C=c \alpha_{1}$ and $D=d \alpha_{1}$, and then we know $A \geq 4, B \geq 3, C \geq 2, D>1$ for the same reasons listed in Case V. If we utilize the estimate provided by Luo et al. [11], we get the following:

$$
\begin{aligned}
5 ! P_{5}= & 5 !\left(P_{4}(1)+P_{4}(2)\right) \\
\leq & 5\left[\left(A\left(\frac{1-\alpha_{1}}{2 \alpha_{1}}+1\right)-1\right)\left(B\left(\frac{1-\alpha_{1}}{2 \alpha_{1}}+1\right)-1\right)\right. \\
& \cdot\left(C\left(\frac{1-\alpha_{1}}{2 \alpha_{1}}+1\right)-1\right) \cdot\left(D\left(\frac{1-\alpha_{1}}{2 \alpha_{1}}+1\right)-1\right) \\
& -\left(D\left(\frac{1-\alpha_{1}}{2 \alpha_{1}}+1\right)-1\right)+D\left(\frac{1-\alpha_{1}}{2 \alpha_{1}}+1\right) \\
& +\left(D\left(\frac{1-\alpha_{1}}{2 \alpha_{1}}+1\right)-1\right)\left(D\left(\frac{1-\alpha_{1}}{2 \alpha_{1}}+1\right)-2\right)
\end{aligned}
$$




$$
\begin{aligned}
& \times\left(D\left(\frac{1-\alpha_{1}}{2 \alpha_{1}}+1\right)-3\right)+(A-1)(B-1)(C-1)(D-1) \\
& -(D-1)^{4}+D(D-1)(D-2)(D-3) .
\end{aligned}
$$

It is sufficient to show that the RHS of the inequality above is strictly less than the RHS of Theorem 2.3. Let $\Delta_{4}$ be the difference between the latter, substituting $a=\frac{A}{\alpha_{1}}, b=\frac{B}{\alpha_{1}}, c=\frac{C}{\alpha_{1}}, d=\frac{D}{\alpha_{1}}, e=\frac{2}{1-\alpha_{1}}, \nu=3$ and the former.

$$
\begin{aligned}
\Delta_{4}= & \frac{1}{16\left(1-\alpha_{1}\right) \alpha_{1}^{4}}\left(11 A B C D-\alpha_{1}(6 A B C+6 A B D+6 A C D\right. \\
& \left.+6 B C D-A B C D \alpha_{1}-20 D^{3}\right) \\
& -\alpha_{1}^{2}(4 A B+4 A C+4 B C-4 A B C+4 A D+4 B D-4 A B D \\
& +4 C D-4 A C D-4 B C D+10 A B C D+100 D^{2}-40 D^{3} \\
& +\alpha_{1}^{3}(24 A+24 B-4 A B+24 C-4 A C-4 B C+104 D-4 A D \\
& \left.-4 B D-4 C D+10 A B C D-100 D^{2}\right) \\
& +\alpha_{1}^{4}(-496+64 A+64 B-60 A B+64 C-60 A C-60 B C+60 A B C \\
& +224 D-60 A D-60 B D+60 A B D-60 C D+60 A C D+60 B C D \\
& \left.-65 A B C D-300 D^{2}+120 D^{3}\right) \\
& +\alpha_{1}^{5}(528-120 A-120 B+100 A B-120 C+100 A C+100 B C \\
& -90 A B C-360 D+100 A D+100 B D-90 A B D+100 C D-90 A C D \\
& \left.\left.-90 B C D+85 A B C D+500 D^{2}-180 D^{3}\right)\right) . \\
= & \frac{1}{16\left(1-\alpha_{1}\right) \alpha_{1}^{4}} \Delta_{5} .
\end{aligned}
$$

Now, we apply the partial differentiation test to $\Delta_{5}$ on the domain $A \geq 4$, $B \geq 3, C \geq 2, D \geq 1$ and $\alpha_{1} \in\left(0, \frac{1}{3}\right]$.

$$
\frac{\partial^{4} \Delta_{5}}{\partial A \partial B \partial C \partial D}=11+\alpha_{1}-10 \alpha_{1}^{2}+10 \alpha_{1}^{3}-65 \alpha_{1}^{4}+85 \alpha_{1}^{5}>0
$$

for $\alpha_{1} \in(0,1)$, so $\frac{\partial^{3} \Delta_{5}}{\partial A \partial B \partial C}$ is an increasing function of $D$ on $D \geq 1$ and $\alpha_{1} \in(0,1)$ with its minimum at $D=1$.

$$
\left.\frac{\partial^{3} \Delta_{5}}{\partial A \partial B \partial C}\right|_{D=1}=11-5 \alpha_{1}-6 \alpha_{1}^{2}+10 \alpha_{1}^{3}-5 \alpha_{1}^{4}-5 \alpha_{1}^{5}>0
$$

so $\frac{\partial^{3} \Delta_{5}}{\partial A \partial B \partial C}>0$ for $D \geq 1$ and $\alpha_{1} \in(0,1)$. Because $\frac{\partial^{2} \Delta_{5}}{\partial A \partial B}$ is symmetric with respect to $C$ and $D, \frac{\partial^{3} \Delta_{5}}{\partial A \partial B \partial D}>0$ for $C \geq 1$ and $\alpha_{1} \in(0,1)$, and $\frac{\partial^{2} \Delta_{5}}{\partial A \partial B}$ is 
an increasing function of $C$ and $D$ for $C \geq D \geq 1$ and $\alpha_{1} \in(0,1)$ with its minimum at $C=D=1$.

$$
\left.\frac{\partial^{2} \Delta_{5}}{\partial A \partial B}\right|_{C=D=1}=\left(\alpha_{1}-1\right)^{2}\left(1+\alpha_{1}\right)\left(11+5 \alpha_{1}^{2}\right)>0
$$

for $\alpha_{1} \in(0,1)$, so $\frac{\partial^{2} \Delta_{5}}{\partial A \partial B}>0$ on the interval $C \geq 1, D \geq 1$ and $\alpha_{1} \in(0,1)$. Because $\frac{\partial \Delta_{5}}{\partial A}$ is symmetric with respect to $B, C$ and $D, \frac{\partial^{2} \Delta_{5}}{\partial A \partial C}>0$ on the interval $B \geq 1, D \geq 1$ and $\alpha_{1} \in(0,1)$, and $\frac{\partial^{2} \Delta_{5}}{\partial A \partial D}>0$ on the interval $B \geq 1$, $C \geq 1$ and $\alpha_{1} \in(0,1)$. Then, we know that $\frac{\partial \Delta_{5}}{\partial A}$ is an increasing function of $B, C$ and $D$ for $B \geq C \geq D \geq 1$ and $\alpha_{1} \in(0,1)$ with its minimum at $B=C=D=1$.

$$
\left.\frac{\partial \Delta_{5}}{\partial A}\right|_{B=C=D=1}=-\left(\alpha_{1}-1\right)^{3}\left(\alpha_{1}+1\right)\left(11+5 \alpha_{1}\right)>0
$$

for $\alpha_{1} \in\left(0, \frac{3}{5}\right)$, so $\frac{\partial \Delta_{5}}{\partial A}>0$ on the interval $B \geq 1, C \geq 1, D \geq 1$ and $\alpha_{1} \in$ $\left(0, \frac{3}{5}\right)$. Due to the fact that $\Delta_{5}$ is symmetric with respect to $A, B$ and $C$, we also have $\frac{\partial \Delta_{5}}{\partial B}>0$ on the interval $A \geq 1, C \geq 1, D \geq 1, \alpha_{1} \in\left(0, \frac{3}{5}\right)$ and $\frac{\partial \Delta_{5}}{\partial C}>0$ on the interval $A \geq 1, B \geq 1, D \geq 1, \alpha_{1} \in\left(0, \frac{3}{5}\right)$. In the meantime,

$$
\frac{\partial^{3} \Delta_{5}}{\partial D^{3}}=120\left(1-\alpha_{1}\right) \alpha_{1}\left(1+3 \alpha_{1}\right)\left(1+3 \alpha_{1}^{2}\right)>0
$$

for $\alpha_{1} \in(0,1)$, so $\frac{\partial^{2} \Delta_{5}}{\partial D^{2}}$ is an increasing function of $D$ for $D \geq 1$ and $\alpha_{1} \in$ $(0,1)$ with its minimum at $D=1$.

$$
\left.\frac{\partial^{2} \Delta_{5}}{\partial D^{2}}\right|_{D=1}=40 \alpha_{1}\left(1-\alpha_{1}\right)\left(3+4 \alpha_{1}-\alpha_{1}^{2}+2 \alpha_{1}^{3}\right)>0
$$

for $\alpha_{1} \in(0,1)$, which means $\frac{\partial^{2} \Delta_{5}}{\partial D^{2}}>0$ for $D \geq 1$ and $\alpha_{1} \in(0,1)$ hence $\frac{\partial \Delta_{5}}{\partial D}$ is an increasing function of $A, B, C$ and $D$, on the interval $A \geq B \geq C \geq$ $D \geq 1, \alpha_{1} \in(0,1)$ with its minimum at $A=B=C=D=1$.

$$
\left.\frac{\partial \Delta_{5}}{\partial D}\right|_{A=4, B=3, C=2, D=1}=4\left(66-18 \alpha_{1}-63 \alpha_{1}^{2}+27 \alpha_{1}^{3}-139 \alpha_{1}^{4}+175 \alpha_{1}^{5}\right)>0
$$

for $\alpha_{1} \in(0,1)$, and thus $\frac{\partial \Delta_{5}}{\partial D}>0$ for $A \geq 4, B \geq 3, C \geq 2, D \geq 1$ and $\alpha_{1} \in$ $(0,1]$. Hence, $\Delta_{5}$ is an increasing function of $A, B, C, D$ for $A \geq 4, B \geq 3$, 
$C \geq 2, D \geq 1$ and $\alpha_{1} \in(0,1]$ with its minimum at $A=4, B=3, C=2$, $D=1$.

$$
\left.\Delta_{5}\right|_{A=4, B=3, C=2, D=1}=8\left(\alpha_{1}-1\right)\left(-33-\alpha_{1}+29 \alpha_{1}^{2}-11 \alpha_{1}^{3}+56 \alpha_{1}^{4}\right) \geq 0
$$

for $\alpha_{1} \in\left(0, \frac{3}{5}\right), \Delta_{5}>0$ for $A \geq 4, B \geq 3, C \geq 2, D \geq 1$ and $\alpha_{1} \in\left(0, \frac{3}{5}\right)$, thus ending this proof.

\subsection{Case III}

Theorem 2.4. Let $a \geq b \geq c \geq d \geq e$ and $e \in(3,4]$ be real numbers. Consider $\frac{x}{a}+\frac{y}{b}+\frac{z}{c}+\frac{v}{d}+\frac{w}{e} \leq 1$. Let $P_{5}$ be the number of positive integral solutions of $\frac{x}{a}+\frac{y}{b}+\frac{z}{c}+\frac{v}{d}+\frac{w}{e} \leq 1$, i.e., $P_{5}=\#\left\{(x, y, z, v, w) \in \mathbb{Z}_{+}^{5}: \frac{x}{a}+\frac{y}{b}+\right.$ $\left.\frac{z}{c}+\frac{v}{d}+\frac{w}{e} \leq 1\right\}$. Define $\mu=(a-1)(b-1)(c-1)(d-1)(e-1)$. Then, if $P_{5}>0$,

$$
\begin{aligned}
120 P_{5} & \leq \mu-\left.\left(5 v^{4}-25 v^{3}+40 v^{2}-19 v-1\right)\right|_{v=4} \\
& =(a-1)(b-1)(c-1)(d-1)(e-1)-(243)
\end{aligned}
$$

with equality if and only if $a=b=c=d=e=$ integer.

Proof. In this case, there are four levels that might contain positive integral solutions: $k=1, k=2, k=3$ and $k=4$. When $k=4$, the defining inequality of the five-dimensional simplex becomes $\frac{x}{a}+\frac{y}{b}+\frac{z}{c}+\frac{v}{d}+\frac{4}{e} \leq 1$, which has no positive integral solutions. Because $P_{5}>0$, we know $k=1$ must have points, but the nature of the levels $k=2$ and $k=3$ are unknown, and therefore, we have to consider three different subcases:

(a) $P_{4}(3)=P_{4}(2)=0$,

(b) $P_{4}(3)=0, P_{4}(2)>0$,

(c) $P_{4}(3)>0, P_{4}(2)>0$.

In subcase (a), we will apply the GLY conjecture for $n=4$ on the level $k=1$ to estimate $P_{4}(1)$. This estimate is given by the expression below, which is also present as Theorem 2.1 of [8]:

$$
4 ! P_{4} \leq a b c d-\frac{3}{2}(a b c+a b d+a c d+b c d)+\frac{11}{3}(a b+a c+b c)-2(a+b+c),
$$

as long as $a \geq b \geq c \geq d \geq 2$ and $P_{4}>0$. 
Let $\alpha$ be defined by $\frac{1}{a}+\frac{1}{b}+\frac{1}{c}+\frac{1}{d} \leq 1-\frac{1}{e} \triangleq \alpha, \alpha \in\left(\frac{2}{3}, \frac{3}{4}\right]$ since $e \in(3,4]$, and let $A=a \alpha, B=b \alpha, C=c \alpha$ and $D=d \alpha$. Then, the following restrictions apply:

$$
A \geq 4, B \geq 3, C \geq D \geq \frac{\alpha}{1-\alpha}>2 .
$$

We can apply the GLY conjecture to the above region because $D \geq \frac{\alpha}{1-\alpha} \in$ $(2,3]$, satisfying the condition of Theorem 2.1 from [8] that the smallest weight has to be greater than or equal to 2 .

$$
\begin{aligned}
5 ! P_{4} \leq & 5\left[A B C D-\frac{3}{2}(A B C+A B D+A C D+B C D)\right. \\
& \left.+\frac{11}{3}(A B+A C+B C)-2(A+B+C)\right] .
\end{aligned}
$$

Let $\Delta_{6}$ now be defined as the difference by subtracting the RHS of the above inequality from the RHS of Theorem 2.4.

$$
\begin{aligned}
\Delta_{6}= & \frac{1}{6(1-\alpha) \alpha^{3}}((1464+110 A C+110 B C-60 A-60 B-60 C \\
& -45 A B C+30 A B C D-45 A B D-45 A C D-45 B C D+110 A B) \alpha^{4} \\
& +(-110 A C-110 B C-110 A B-1458+45 A B C+54 A+54 B \\
& +45 B C D+45 A B D+45 A C D-6 D+54 C-30 A B C D) \alpha^{3} \\
& +(6 B C+6 A D+6 B D+6 C D+6 A C+6 A B) \alpha^{2} \\
& +(-6 A B C-6 A B D-6 A C D-6 B C D) \alpha+6 A B C D) \\
= & \frac{1}{6(1-\alpha) \alpha^{3}} \Delta_{7} .
\end{aligned}
$$

Then, we only need to show that $\Delta_{7}>0$ by the partial differentiation test.

$$
\frac{\partial^{4} \Delta_{7}}{\partial A \partial B \partial C \partial D}=6-30 \alpha^{3}+30 \alpha^{4}>0
$$

for all $\alpha \in\left(\frac{2}{3}, \frac{3}{4}\right]$. Then, $\frac{\partial^{3} \Delta_{7}}{\partial A \partial B \partial C}$ is an increasing function of $D$ on $D \geq 1$, $\alpha \in\left(\frac{2}{3}, \frac{3}{4}\right]$ with a minimum at $D=2$.

$$
\left.\frac{\partial^{3} \Delta_{7}}{\partial A \partial B \partial C}\right|_{D=2}=15 \alpha^{4}-15 \alpha^{3}-6 \alpha+12>0
$$

for all $\alpha \in\left(\frac{2}{3}, \frac{3}{4}\right]$, so $\frac{\partial^{3} \Delta_{7}}{\partial A \partial B \partial C}>0$ for all $D \geq 2, \alpha \in\left(\frac{2}{3}, \frac{3}{4}\right]$, and because $\frac{\partial^{2} \Delta_{7}}{\partial A \partial B}$ is symmetric with respect to $C$ and $D$, we also know $\frac{\partial^{3} \Delta_{7}}{\partial A \partial B \partial D}>0$ for all $C \geq$ $2, \alpha \in\left(\frac{2}{3}, \frac{3}{4}\right]$. (From symmetry, we also know that $\frac{\partial^{3} \Delta_{7}}{\partial A \partial C \partial D}>0$ for all $B \geq 2$, 
$\alpha \in\left(\frac{2}{3}, \frac{3}{4}\right]$, a fact that will come in handy later in the proof.) Therefore, $\frac{\partial^{2} \Delta_{7}}{\partial A \partial B}$ is an increasing function with respect to $C$ and $D$ for all $C \geq \frac{\alpha}{1-\alpha}$, $D \geq \frac{\alpha}{1-\alpha}$ and $\alpha \in\left(\frac{2}{3}, \frac{3}{4}\right]$ with a minimum at $C=D \geq \frac{\alpha}{1-\alpha}>2$.

$$
\left.\frac{\partial^{2} \Delta_{7}}{\partial A \partial B}\right|_{C=D=2}=50 \alpha^{4}-50 \alpha^{3}+6 \alpha^{2}-24 \alpha+24>0
$$

for all $\alpha \in\left(\frac{2}{3}, \frac{3}{4}\right]$. It follows that $\frac{\partial^{2} \Delta_{7}}{\partial A \partial B}>0$ for all $C \geq \frac{\alpha}{1-\alpha}>2, D \geq \frac{\alpha}{1-\alpha}>2$ and $\alpha \in\left(\frac{2}{3}, \frac{3}{4}\right]$, and because $\frac{\partial \Delta_{7}}{\partial A}$ is symmetric with respect to $B$ and $C$, we also know $\frac{\partial^{2} \Delta_{7}}{\partial A \partial C}>0$ for all $B \geq \frac{\alpha}{1-\alpha}>2, D \geq \frac{\alpha}{1-\alpha}>2, \alpha \in\left(\frac{2}{3}, \frac{3}{4}\right]$. We now need to show that $\frac{\partial^{2} \Delta_{7}}{\partial A \partial D}>0$ for all $B \geq \frac{\alpha}{1-\alpha}, C \geq \frac{\alpha}{1-\alpha}, \alpha \in\left(\frac{2}{3}, \frac{3}{4}\right]$; we already know that it is an increasing function on the domain because $\frac{\partial^{3} \Delta_{7}}{\partial A \partial B \partial D}>0$ and $\frac{\partial^{3} \Delta_{7}}{\partial A \partial C \partial D}>0$ for their respective domains so we only need to check the value of $\frac{\partial^{2} \Delta_{7}}{\partial A \partial D}$ at its minimum of $B=3, C=\frac{\alpha}{1-\alpha}>2$.

$$
\left.\frac{\partial^{2} \Delta_{7}}{\partial A \partial D}\right|_{B=3, C=2}=-45 \alpha^{4}+45 \alpha^{3}+6 \alpha^{2}-30 \alpha+36>0
$$

for all $\alpha \in\left(\frac{2}{3}, \frac{3}{4}\right.$ ], so $\frac{\partial^{2} \Delta_{7}}{\partial A \partial D}>0$ on the domain. (By symmetry, we also know $\frac{\partial^{2} \Delta_{7}}{\partial B \partial D}>0$ and $\frac{\partial^{2} \Delta_{7}}{\partial C \partial D}>0$ on their respective domains, which will help later in the proof.) Therefore, $\frac{\partial \Delta_{7}}{\partial A}$ is an increasing function with respect to $B, C$, $D$ for all $B \geq 3, C \geq \frac{\alpha}{1-\alpha}, D \geq \frac{\alpha}{1-\alpha}, \alpha \in\left(\frac{2}{3}, \frac{3}{4}\right]$ with a minimum at $B=3$, $C=D=\frac{\alpha}{1-\alpha}>2$.

$$
\left.\frac{\partial \Delta_{7}}{\partial A}\right|_{B=3, C=D=2}=130 \alpha^{4}-136 \alpha^{3}+42 \alpha^{2}-96 \alpha+72>0
$$

for all $\alpha \in\left(\frac{2}{3}, \frac{3}{4}\right]$ so $\frac{\partial \Delta_{7}}{\partial A}>0$ for $B \geq 3, C \geq \frac{\alpha}{1-\alpha}, D \geq \frac{\alpha}{1-\alpha}, \alpha \in\left(\frac{2}{3}, \frac{3}{4}\right]$. By the property that $\Delta_{7}$ is symmetric with respect to $A, B, C$, we also have $\frac{\partial \Delta_{7}}{\partial B}>0$ for $A \geq 3, C \geq \frac{\alpha}{1-\alpha}, D \geq \frac{\alpha}{1-\alpha}, \alpha \in\left(\frac{2}{3}, \frac{3}{4}\right]$ and $\frac{\partial \Delta_{7}}{\partial C}>0$ for $A \geq 3, B \geq \frac{\alpha}{1-\alpha}$, $D \geq \frac{\alpha}{1-\alpha}, \alpha \in\left(\frac{2}{3}, \frac{3}{4}\right]$, and therefore, $\Delta_{7}$ is an increasing function with respect to $A, B, C$. Meanwhile, because we know $\frac{\partial^{2} \Delta_{7}}{\partial A \partial D}>0, \frac{\partial^{2} \Delta_{7}}{\partial B \partial D}>0, \frac{\partial^{2} \Delta_{7}}{\partial C \partial D}>0$ on their respective domains, we know that $\frac{\partial \Delta_{7}}{\partial D}$ is an increasing function with respect to $A, B, C$ for $A \geq 3, B \geq 3, C \geq \frac{\alpha}{1-\alpha}, \alpha \in\left(\frac{2}{3}, \frac{3}{4}\right]$ with its minimum at $A=4, B=3, C=\frac{\alpha}{1-\alpha}>2$.

$$
\left.\frac{\partial \Delta_{7}}{\partial D}\right|_{A=4, B=3, C=2}=-450 \alpha^{4}+444 \alpha^{3}+54 \alpha^{2}-156 \alpha+144>0
$$


for all $\alpha \in\left(\frac{2}{3}, \frac{3}{4}\right]$ so $\frac{\partial \Delta_{7}}{\partial D}>0$ for $A \geq 4, B \geq 3, C \geq \frac{\alpha}{1-\alpha}, \alpha \in\left(\frac{2}{3}, \frac{3}{4}\right]$. It follows that $\Delta_{7}$ is an increasing function with respect to $D$, too. We separate the proof over the region considered into the following cases.

Case (1). $A \geq 4, B \geq 3, C \geq D \geq 2$.76. It's easy to verify $\Delta_{7} \geq 0$ in this subcase.

Case (2). $2.76 \geq D \geq \frac{\alpha}{1-\alpha}$. Hence $\frac{1}{A}+\frac{1}{B}+\frac{1}{C} \leq 1-\frac{1}{D} \leq 1-\frac{1}{2.76}=\frac{44}{69}$. $\frac{1}{\frac{44}{69} A}+\frac{1}{\frac{44}{69} B}+\frac{1}{\frac{44}{69} C} \leq 1$. Hence $\frac{44}{69} A \geq 3, \frac{44}{69} B \geq 2$. We obtain $A \geq \frac{207}{44}, B \geq \frac{69}{22}$. It is easy to verify $\Delta_{7} \geq 0$ for $A \geq \frac{207}{44}, B \geq C \geq 3.41$ and $2.76 \geq D \geq \frac{\alpha}{1-\alpha}$. Case (3). $2.76 \geq D \geq \frac{\alpha}{1-\alpha}$, and $D \leq C \leq 3.41$.

Hence $\frac{1}{A}+\frac{1}{B} \leq 1-\frac{1}{C}-\frac{1}{D} \leq 1-\frac{1}{2.76}-\frac{1}{3.41}=\frac{8104}{23529}$. Hence $\frac{8104}{23529} A \geq 2$. We obtain $A \geq \frac{23529}{4052}$.

It's easy to verify $\Delta_{7} \geq 0$ for $A \geq \frac{23529}{4052}, B \geq 5.59,2.76 \geq D \geq \frac{\alpha}{1-\alpha}$ and $D \leq$ $C \leq 3.41$.

Case (4). When $2.76 \geq D \geq \frac{\alpha}{1-\alpha}, D \leq C \leq 3.41$ and $5.59 \geq B \geq \frac{69}{22}$. $\frac{1}{A} \leq 1-\frac{1}{B}-\frac{1}{C}-\frac{1}{D} \leq 1-\frac{1}{5.59}-\frac{1}{3.41}-\frac{1}{2.76}=\frac{2177236}{13152711}$. Hence $A \geq \frac{13152711}{2177236}$. It is easy to verify $\Delta_{7} \geq 0$ for $A \geq 11.5, \frac{69}{22} \leq B \leq 5.59,2.76 \geq D \geq \frac{\alpha}{1-\alpha}$ and $D \leq C \leq 3.41$.

Case (5). We still need to consider $11.5 \geq A \geq \frac{13152711}{2177236}, 5.59 \geq B \geq \frac{69}{22}$, $2.76 \geq D \geq \frac{\alpha}{1-\alpha}$, and $D \leq C \leq 3.41$.

From $\frac{1}{A}+\frac{1}{B}+\frac{1}{C}+\frac{1}{D} \leq 1$ we have the following:

$\frac{1}{D} \leq 1-\frac{1}{A}-\frac{1}{B}-\frac{1}{C} \leq 1-\frac{1}{11.5}-\frac{1}{5.59}-\frac{1}{3.41}=\frac{1932999}{4384237}$ Hence $D \geq \frac{4384237}{1932999}$.

$\frac{1}{B} \leq 1-\frac{1}{A}-\frac{1}{C}-\frac{1}{D} \leq 1-\frac{1}{11.5}-\frac{1}{3.41}-\frac{1}{2.76}=\frac{6058}{23529}$. Hence $B \geq \frac{23529}{6058}$.

$\frac{1}{C} \leq 1-\frac{1}{A}-\frac{1}{B}-\frac{1}{D} \leq 1-\frac{1}{11.5}-\frac{1}{5.59}-\frac{1}{2.76}=\frac{14342}{38571}$. Hence $C \geq \frac{38571}{14342}$.

It is easy to verify $\Delta_{7} \geq 0$ for $11.5 \geq A \geq \frac{13152711}{2177236}, 5.59 \geq B \geq \frac{23529}{6058}, 3.41 \geq$ $C \geq \frac{38571}{14342}$ and $2.76 \geq D \geq \frac{4384237}{1932999}$. Then, it follows that $\Delta_{7}>0$ for $A \geq$ $4, B \geq 3, C \geq D \geq \frac{\alpha}{1-\alpha}$ and $\alpha \in\left(\frac{2}{3}, \frac{3}{4}\right]$.

For subcase (b), the proof is similar to the proof in subcase $\operatorname{IV}(\mathrm{b})$. Because $P_{4}(2)>0,(1,1,1,1,2)$ is the smallest positive solution for the level $k=2$, and so, let $\alpha_{1}$ be defined by $\frac{1}{a}+\frac{1}{b}+\frac{1}{c}+\frac{1}{d} \leq 1-\frac{2}{e} \triangleq \alpha_{1}, \alpha_{1} \in\left(\frac{1}{3}, \frac{1}{2}\right]$, since $e \in(3,4]$. Let $A=a \alpha_{1}, B=b \alpha_{1}, C=c \alpha_{1}$ and $D=d \alpha_{1}$; then the following restrictions apply:

$$
A \geq 4, B \geq 3, C \geq 2, D \geq \frac{2 \alpha_{1}}{1-\alpha_{1}},
$$

since $D=d \alpha_{1} \geq e \alpha_{1}=\frac{2 \alpha_{1}}{1-\alpha_{1}}$. With $\alpha_{1} \in\left(\frac{1}{3}, \frac{1}{2}\right], \frac{2 \alpha_{1}}{1-\alpha_{1}} \in(1,2]$, so it is sufficient to show $\Delta_{4}-211=\frac{1}{16\left(1-\alpha_{1}\right) \alpha_{1}^{4}}\left(\Delta_{5}-211(16) \alpha_{1}^{4}\left(1-\alpha_{1}\right)\right)>0$, hence to show that $\Delta_{5}-211(16) \alpha_{1}^{4}\left(1-\alpha_{1}\right)>0$ for $A \geq 4, B \geq 3, C \geq 2, D \geq \frac{2 \alpha_{1}}{1-\alpha_{1}}$ and $\alpha_{1} \in\left(\frac{1}{3}, \frac{1}{2}\right]$. Note that the term $-211(16) \alpha_{1}^{4}\left(1-\alpha_{1}\right)$ only affects the 
value of the function at the minimum, and therefore, the proof of this subcase is exactly the same as the one in subcase IV (b) except for the very last calculation, the calculation of the value of the function's minimum.

$$
\begin{aligned}
\Delta_{5} & -\left.211(16) \alpha_{1}^{4}\left(1-\alpha_{1}\right)\right|_{A=4, B=3, C=2, D=\frac{2 \alpha_{1}}{1-\alpha_{1}}} \\
& =\frac{16 \alpha_{1}\left(24-32 \alpha_{1}-6 \alpha_{1}^{2}-183 \alpha_{1}^{3}+565 \alpha_{1}^{4}-597 \alpha_{1}^{5}+389 \alpha_{1}^{6}\right)}{\left(\alpha_{1}-1\right)^{2}}>0,
\end{aligned}
$$

for $\alpha_{1} \in\left(\frac{1}{3}, \frac{1}{2}\right]$. Then, it follows that $\Delta_{5}-211(16) \alpha_{1}^{4}\left(1-\alpha_{1}\right)>0$ for $A \geq 4$, $B \geq 3, C \geq 2, D \geq \frac{2 \alpha_{1}}{1-\alpha_{1}}$ and $\alpha_{1} \in\left(\frac{1}{3}, \frac{1}{2}\right]$.

For subcase (c), $P_{4}(3)>0$, so $(1,1,1,1,3)$ is the smallest positive solution on the level $k=3$. Let $\alpha_{2}$ be defined by $\frac{1}{a}+\frac{1}{b}+\frac{1}{c}+\frac{1}{d} \leq 1-\frac{3}{e} \triangleq \alpha_{2}$, $\alpha_{2} \in\left(0, \frac{1}{4}\right]$, since $e \in(3,4]$, and let $A=a \alpha_{2}, B=b \alpha_{2}, C=c \alpha_{2}$ and $D=d \alpha_{2}$. Also, notice that $A \geq 4, B \geq 3, C \geq 2, D \geq 1$, for the same reasons listed in Case V. Then, from the estimate provided in [11], we know that

$$
\begin{aligned}
5 ! P_{5}= & 5 !\left(P_{4}(1)+P_{4}(2)+P_{4}(3)\right) \\
\leq & 5\left[\left(A \frac{2+\alpha_{2}}{3 \alpha_{2}}-1\right)\left(B \frac{2+\alpha_{2}}{3 \alpha_{2}}-1\right)\left(C \frac{2+\alpha_{2}}{3 \alpha_{2}}-1\right)\right. \\
& \times\left(D \frac{2+\alpha_{2}}{3 \alpha_{2}}-1\right)-\left(D \frac{2+\alpha_{2}}{3 \alpha_{2}}-1\right)+\left(D \frac{2+\alpha_{2}}{3 \alpha_{2}}\right) \\
& \times\left(D \frac{2+\alpha_{2}}{3 \alpha_{2}}-1\right)\left(D \frac{2+\alpha_{2}}{3 \alpha_{2}}-2\right)\left(D \frac{2+\alpha_{2}}{3 \alpha_{2}}-3\right) \\
& +\left(A \frac{1+2 \alpha_{2}}{3 \alpha_{2}}-1\right)\left(B \frac{1+2 \alpha_{2}}{3 \alpha_{2}}-1\right)\left(C \frac{1+2 \alpha_{2}}{3 \alpha_{2}}-1\right) \\
& \times\left(D \frac{1+2 \alpha_{2}}{3 \alpha_{2}}-1\right)-\left(D \frac{1+2 \alpha_{2}}{3 \alpha_{2}}-1\right)+\left(D \frac{1+2 \alpha_{2}}{3 \alpha_{2}}\right) \\
& \times\left(D \frac{1+2 \alpha_{2}}{3 \alpha_{2}}-1\right)\left(D \frac{1+2 \alpha_{2}}{3 \alpha_{2}}-2\right)\left(D \frac{1+2 \alpha_{2}}{3 \alpha_{2}}-3\right) \\
& +(A-1)(B-1)(C-1)(D-1)-(D-1)^{4} \\
& +D(D-1)(D-2)(D-3)] .
\end{aligned}
$$

It is sufficient to show that the RHS of the inequality above is strictly less than the RHS of Theorem 2.4. Let $\Delta_{8}$ be the difference by subtracting the RHS of the inequality above from the RHS of Theorem 2.4, substituting 
$a=\frac{A}{\alpha_{2}}, b=\frac{B}{\alpha_{2}}, c=\frac{C}{\alpha_{2}}, d=\frac{D}{\alpha_{2}}, e=\frac{3}{1-\alpha_{2}}, \nu=4$, and the former.

$$
\begin{aligned}
\Delta_{8}= & \frac{1}{81\left(1-\alpha_{2}\right) \alpha_{2}^{4}}\left(77 A B C D-\alpha_{2}(27 A B C+27 A B D+27 A C D\right. \\
& \left.+27 B C D+34 A B C D-270 D^{3}\right)-\alpha_{2}^{2}(63 A B+63 A C+63 B C \\
& -54 A B C+63 A D+63 B D-54 A B D+63 C D-54 A C D \\
& \left.-54 B C D+40 A B C D+1125 D^{2}-270 D^{3}\right)+\alpha_{2}^{3}(243 A+243 B \\
& -54 A B+243 C-54 A C-54 B C+1053 D-54 A D-54 B D \\
& \left.-54 C D+40 A B C D-675 D^{2}\right)+\alpha_{2}^{4}(324 A-19521+324 B \\
& -270 A B+324 C-270 A C-270 B C+270 A B C+1134 D \\
& -270 A D-270 B D+270 A B D-270 C D+270 A C D \\
& \left.+270 B C D-290 A B C D-1350 D^{2}+540 D^{3}\right)+\alpha_{2}^{5}(19764 \\
& -810 A-810 B+630 A B-810 C+630 A C+630 B C-540 A B C \\
& -2430 D+630 A D+630 B D-540 A B D+630 C D-540 A C D \\
& \left.-540 B C D+490 A B C D+3150 D^{2}-1080 D^{3}\right) \\
= & \frac{1}{81\left(1-\alpha_{2}\right) \alpha_{2}^{4}} \Delta_{9} .
\end{aligned}
$$

Now, we apply the partial differentiation test on $\Delta_{9}$ for $A \geq 4, B \geq 3$, $C \geq 2, D \geq 1$ and $\alpha_{2} \in\left(0, \frac{1}{4}\right]$.

$$
\begin{aligned}
\frac{\partial^{4} \Delta_{9}}{\partial A \partial B \partial C \partial D} & =77-34 \alpha_{2}-40 \alpha_{2}^{2}+40 \alpha_{2}^{3}-290 \alpha_{2}^{4}+490 \alpha_{2}^{5} \\
& >0
\end{aligned}
$$

for $\alpha_{2} \in\left(0, \frac{2}{5}\right)$. It follows that $\frac{\partial^{3} \Delta_{9}}{\partial A \partial B \partial C}$ is an increasing function of $D$ for $D \geq 1$ and $\alpha_{2} \in\left(0, \frac{2}{5}\right)$, with a minimum at $D=1$ :

$$
\begin{aligned}
\left.\frac{\partial^{3} \Delta_{9}}{\partial A \partial B \partial C}\right|_{D=1} & =77-61 \alpha_{2}+14 \alpha_{2}^{2}+40 \alpha_{2}^{3}-20 \alpha_{2}^{4}-50 \alpha_{2}^{5} \\
& >0
\end{aligned}
$$

for $\alpha_{2} \in\left(0, \frac{2}{5}\right)$, so $\frac{\partial^{3} \Delta_{9}}{\partial A \partial B \partial C}>0$ for $D \geq 1$ and $\alpha_{2} \in\left(0, \frac{2}{5}\right)$. Because $\frac{\partial^{2} \Delta_{9}}{\partial A \partial B}$ is symmetric with respect to $C$ and $D$, we also know that $\frac{\partial^{3} \Delta_{9}}{\partial A \partial B \partial D}>0$ for $C \geq 1$ and $\alpha_{2} \in\left(0, \frac{2}{5}\right)$, and thus, $\frac{\partial^{2} \Delta_{9}}{\partial A \partial B}$ is an increasing function of $C$ and $D$ 
for $C \geq D \geq 1$ and $\alpha_{2} \in\left(0, \frac{2}{5}\right)$ with a minimum at $C=D=1$.

$$
\begin{aligned}
\left.\frac{\partial^{2} \Delta_{9}}{\partial A \partial B}\right|_{C=D=1} & =77-88 \alpha_{2}+5 \alpha_{2}^{2}-14 \alpha_{2}^{3}-20 \alpha_{2}^{4}+40 \alpha_{2}^{5} \\
& >0
\end{aligned}
$$

for $\alpha_{2} \in\left(0, \frac{2}{5}\right)$, so $\frac{\partial^{2} \Delta_{9}}{\partial A \partial B}>0$ for $C \geq D \geq 1$ and $\alpha_{2} \in\left(0, \frac{2}{5}\right)$. Due to the fact that $\frac{\partial \Delta_{9}}{\partial A}$ is symmetric with respect to $B, C$ and $D$, we also know that $\frac{\partial^{2} \Delta_{9}}{\partial A \partial C}>0$ for $B \geq D \geq 1, \alpha_{2} \in\left(0, \frac{2}{5}\right)$ and $\frac{\partial^{2} \Delta_{9}}{\partial A \partial D}>0$ for $B \geq C \geq 1, \alpha_{2} \in$ $\left(0, \frac{2}{5}\right)$. Thus, $\frac{\partial \Delta_{9}}{\partial A}$ is an increasing function of $B, C$ and $D$ for $B \geq C \geq D \geq 1$ and $\alpha_{2} \in\left(0, \frac{2}{5}\right)$ with a minimum at $B=C=D=1$.

$$
\begin{aligned}
\left.\frac{\partial \Delta_{9}}{\partial A}\right|_{B=C=D=1} & =\left(1-\alpha_{2}\right)^{3}\left(77+116 \alpha_{2}+50 \alpha_{2}^{2}\right) \\
& >0
\end{aligned}
$$

for $\alpha_{2} \in\left(0, \frac{2}{5}\right)$, so $\frac{\partial \Delta_{9}}{\partial A}>0$ for $B \geq C \geq D \geq 1$ and $\alpha_{2} \in\left(0, \frac{2}{5}\right)$. Because $\Delta_{9}$ is symmetric with respect to $A, B$ and $C$, we also know that $\frac{\partial \Delta_{9}}{\partial B}>0$ for $A \geq C \geq D \geq 1, \alpha_{2} \in\left(0, \frac{2}{5}\right)$ and $\frac{\partial \Delta_{9}}{\partial C}>0$ for $A \geq B \geq D \geq 1, \alpha_{2} \in\left(0, \frac{2}{5}\right)$. In the meantime, we also have

$$
\begin{aligned}
\frac{\partial^{3} \Delta_{9}}{\partial D^{3}} & =1620\left(1-\alpha_{2}\right)\left(\alpha_{2}\right)\left(1+2 \alpha_{2}\right)\left(1+2 \alpha_{2}^{2}\right) \\
& >0
\end{aligned}
$$

for $\alpha_{2} \in\left(0, \frac{2}{5}\right)$, so $\frac{\partial^{2} \Delta_{9}}{\partial D^{2}}$ is an increasing function of $D$ for $D \geq 1$ and $\alpha_{2} \in$ $\left(0, \frac{2}{5}\right)$, with a minimum at $D=1$.

$$
\begin{aligned}
\left.\frac{\partial^{2} \Delta_{9}}{\partial D^{2}}\right|_{D=1} & =90\left(1-\alpha_{2}\right)\left(\alpha_{2}\right)\left(18+11 \alpha_{2}-4 \alpha_{2}^{2}+2 \alpha_{2}^{3}\right) \\
& >0
\end{aligned}
$$

for $\alpha_{2} \in\left(0, \frac{2}{5}\right)$, so $\frac{\partial \Delta_{9}}{\partial D}$ is an increasing function of $D$ for $D \geq 1$ and $\alpha_{2} \in$ $\left(0, \frac{2}{5}\right)$; furthermore, because $\frac{\partial \Delta_{9}}{\partial D}$ is symmetric with respect to $A, B, C$ and $D$, it is also an increasing function of $A, B$ and $C$ for $A \geq B \geq C \geq D \geq 1$, $\alpha_{2} \in\left(0, \frac{2}{5}\right)$, with a minimum at $A=B=C=D=1$.

$$
\begin{aligned}
\left.\frac{\partial \Delta_{9}}{\partial D}\right|_{A=B=C=D=1} & =\left(1-\alpha_{2}\right)\left(77+772 \alpha_{2}-735 \alpha_{2}^{2}-1154 \alpha_{2}^{3}-1390 \alpha_{2}^{5}\right) \\
& >0
\end{aligned}
$$


for $\alpha_{2} \in\left(0, \frac{2}{5}\right)$, so it follows that $\Delta_{9}$ is an increasing function of $D$ for $D \geq 1$ and $\alpha_{2} \in\left(0, \frac{2}{5}\right)$. We also know that $\Delta_{9}$ is an increasing function of $A, B$ and $C$ for $A \geq B \geq C \geq 1$ and $\alpha_{2} \in\left(0, \frac{2}{5}\right)$, and therefore we only need to check if $\Delta_{9}>0$ at the minimum $A=4, B=3, C=2, D=1$.

$$
\begin{aligned}
\left.\Delta_{9}\right|_{A=4, B=3, C=2, D=1} & =3\left(1-\alpha_{2}\right)\left(616-16 \alpha_{2}-456 \alpha_{2}^{2}+89 \alpha_{2}^{3}-6308 \alpha_{2}^{4}\right) \\
& >0
\end{aligned}
$$

for $\alpha_{2} \in\left(0, \frac{1}{4}\right]$, so $\Delta_{9}>0$ for $A \geq 4, B \geq 3, C \geq 2, D \geq 1$ and $\alpha_{2} \in\left(0, \frac{1}{4}\right]$.

\subsection{Case II}

We will now analyze the case that occurs when $e>4, e \notin \mathbb{Z}_{+}$. We must also keep in mind the hypothesis that the non-integral portion of $e, \beta$ has to be either $\frac{e}{a}, \frac{e}{b}, \frac{e}{c}$, or $\frac{e}{d}$, which is shown by Theorem 2.5 below.

Theorem 2.5. A polynomial $f\left(z_{1}, \ldots, z_{n}\right)$ is weighted homogeneous of type $\left(w_{1}, \ldots, w_{n}\right)$ and has an isolated singularity at the origin. Suppose $w_{i_{1}} \geq$ $w_{i_{2}} \geq \cdots \geq w_{i_{n}}$ and $w_{i_{n}}$ is not an integer, where $\left\{i_{1} . i_{2}, \ldots, i_{n}\right\}=\{1,2, \ldots$, $n\}$. Let $w_{i_{n}}=\left[w_{i_{n}}\right]+\beta$ with $0<\beta<1$. Then, there exists $a j \in\{1, \ldots, n-$ 1) such that $\beta=\frac{w_{i_{n}}}{w_{i_{j}}}$.

Proof. Case 1: $w_{i_{1}}=w_{i_{2}}=\cdots=w_{i_{n}}$. This means $f$ is homogeneous, which implies that $w_{i_{1}}=\cdots=w_{i_{n}}$ are integers, and that contradicts our initial assumption that $w_{i_{n}}$ is not an integer.

Case 2: $\quad w_{i_{1}} \geq w_{i_{2}} \geq \cdots \geq w_{i_{n-1}}>w_{i_{n}}$. Write $f=\sum x_{i_{1}}^{\alpha_{1}} x_{i_{2}}^{\alpha_{2}} \ldots x_{i_{n}}^{\alpha_{n}}$, where $w t\left(x_{i_{j}}\right)=w_{i_{j}}$ and $1 \leq j \leq n$. Then, $\frac{\alpha_{1}}{w_{i_{1}}}+\cdots+\frac{\alpha_{n-1}}{w_{i_{n-1}}}+\frac{\alpha_{n}}{w_{i_{n}}}=1$. Since $w_{i_{n}}$ is not an integer, $x_{i_{n}}^{\alpha_{n}} \notin \operatorname{supp}(f)$. Because $f$ has an isolated singularity at the origin and by the lemma in [17], there exists a $j \in\{1,2, \ldots, n-1\}$ such that $x_{i_{j}} \cdot x_{i_{n}}^{\alpha_{n}} \in \operatorname{supp}(f)$. Therefore,

$$
\begin{aligned}
\frac{1}{w_{i_{j}}}+\frac{\alpha_{n}}{w_{i_{n}}} & =1 \\
\Rightarrow \quad \frac{w_{i_{n}}}{w_{i_{j}}} & =w_{i_{n}}-\alpha_{n} \\
\Rightarrow \quad \beta & =\frac{w_{i_{n}}}{w_{i_{j}}} .
\end{aligned}
$$

Case 3: $w_{i_{1}} \geq \cdots \geq w_{i_{j}}>w_{i_{j+1}}=\cdots=w_{i_{n}}$, where $1 \geq j \geq n-2$. 
Write $f=\sum x_{i_{1}}^{\alpha_{1}} x_{i_{2}}^{\alpha_{2}} \ldots x_{i_{n}}^{\alpha_{n}}$, where $w t\left(x_{i_{j}}\right)=w_{i_{j}}$ and $1 \leq j \leq n$. Then, $\frac{\alpha_{1}}{w_{i_{1}}}+\cdots+\frac{\alpha_{i_{j}}}{w_{i_{j}}}+\frac{\alpha_{j+1}+\cdots+\alpha_{n}}{w_{i_{n}}}=1$. Since $w_{i_{n}}$ is not an integer, $x_{i_{j+1}}^{\alpha_{j+1}} x_{i_{j+2}}^{\alpha_{j+2}} \cdots$ $x_{i_{n}}^{\alpha_{n}} \notin \operatorname{supp}(f)$, and in particular, $x_{i_{n}}^{\alpha_{n}} \notin \operatorname{supp}(f)$. Because $f$ has an isolated singularity at the origin and by the lemma in [17], there exists a $t$ such that $x_{i_{t}} x_{i_{n}}^{\alpha_{n}} \in \operatorname{supp}(f)$, where $1 \leq t \leq j$. Therefore,

$$
\begin{aligned}
\frac{1}{w_{i_{t}}}+\frac{\alpha_{n}}{w_{i_{n}}} & =1 \\
\Rightarrow \quad \frac{w_{i_{n}}}{w_{i_{t}}} & =w_{i_{n}}-\alpha_{n} \\
\Rightarrow \quad \beta & =\frac{w_{i_{n}}}{w_{i_{t}}},
\end{aligned}
$$

and therefore the theorem is proved.

Theorem 2.6. Let $a \geq b \geq c \geq d \geq e>4$ be real numbers, where $e$ is not an integer. Consider $\frac{x}{a}+\frac{y}{b}+\frac{z}{c}+\frac{v}{d}+\frac{w}{e} \leq 1$. Let $P_{5}$ be the number of positive integral solutions of the above inequality; i.e., $P_{5}=\#\{(x, y, z, v, w) \in$ $\left.\mathbb{Z}_{+}^{5}: \frac{x}{a}+\frac{y}{b}+\frac{z}{c}+\frac{v}{d}+\frac{w}{e} \leq 1\right\}$. Suppose $e$ is not an integer and $e=[e]+\beta$ where $\beta$ is either $\frac{e}{a}, \frac{e}{b}, \frac{e}{c}$ or $\frac{e}{d}$. Define $\mu=(a-1)(b-1)(c-1)(d-1)(e-$ 1). Then,

$$
\begin{aligned}
120 P_{5}< & \mu-\left.\left(5 v^{4}-25 v^{3}+40 v^{2}-19 v-1\right)\right|_{v=e-\beta+1} \\
= & a b c d e-(a b c d+a b c e+a b d e+a c d e+b c d e) \\
& +(a b c+a b d+a b e+a c d+a c e+a d e+b c d+b c e+b d e+c d e) \\
& -(a b+a c+a d+a e+b c+b d+b e+c d+c e+d e) \\
& +(a+b+c+d+e)-1-5 e^{4}+5 e^{3}+5 e^{2}-6 e-1 \\
& +\beta\left(20 e^{3}-15 e^{2}-10 e+6\right)-\beta^{2}\left(30 e^{2}+15 e+5\right) \\
& +\beta^{3}(20 e-5)-5 \beta^{4}+1 .
\end{aligned}
$$

Proof. By level $w=k$, we shall mean the intersection of the tetrahedron in Theorem 2.6 with the hyperplane $w=k$. The points within this intersection for level $w=k$ are in following four-dimensional tetrahedron

$$
\frac{x}{a\left(1-\frac{k}{e}\right)}+\frac{y}{b\left(1-\frac{k}{e}\right)}+\frac{z}{c\left(1-\frac{k}{e}\right)}+\frac{v}{d\left(1-\frac{k}{e}\right)} \leq 1
$$

we can use GLY sharp upper estimate to bound the number of positive integral solutions at level $w=k$ if $d\left(1-\frac{k}{e}\right) \geq 3$ or $d\left(1-\frac{k}{e}\right) \geq 2$ with the 
condition that there exist positive integral solutions at this level. By summing these upper bounds in various levels, this leads us to consider the following function:

$$
\begin{aligned}
& g(a, b, c, d, e, n) \\
& =5 \sum_{k=1}^{n}\left[a b c d\left(1-\frac{k}{e}\right)^{4}-\frac{3}{2}(a b c+a b d+a c d+b c d)\left(1-\frac{k}{e}\right)^{3}\right. \\
& \left.\quad+\frac{11}{3}(a b+b c+a c)\left(1-\frac{k}{e}\right)^{2}-2(a+b+c)\left(1-\frac{k}{e}\right)\right]
\end{aligned}
$$

Define $S_{5}=a b c d e, S_{4}=a b c d, S_{3}=a b c+a b d+a c d+b c d, S_{2}=a b+a c+$ $b c$ and $S_{1}=a+b+c$. Then we have

$$
\begin{aligned}
g(a, b, & , d, e, n) \\
= & S_{5}\left(\frac{n}{e}\right)^{5}+\left[S_{4}\left(\frac{1}{2 e^{4}}-\frac{1}{e^{3}}\right)+\frac{3}{8} \frac{S_{3}}{e^{3}}\right] 5 n^{4} \\
& +\left[S_{4}\left(\frac{1}{3 e^{4}}-\frac{2}{e^{3}}+\frac{2}{e^{2}}\right)+3 S_{3}\left(\frac{1}{4 e^{3}}-\frac{1}{2 e^{2}}\right)+\frac{11}{9} \frac{S_{2}}{e^{2}}\right] 5 n^{3} \\
& +\left[S_{4}\left(\frac{-1}{e^{3}}+\frac{3}{e^{2}}-\frac{2}{e}\right)+3 S_{3}\left(\frac{1}{8 e^{3}}-\frac{3}{4 e^{2}}+\frac{3}{4 e}\right)\right. \\
& \left.+11 S_{2}\left(\frac{1}{6 e^{2}}-\frac{1}{3 e}\right)+\frac{S_{1}}{e}\right] 5 n^{2} \\
& +\left[S_{4}\left(\frac{-1}{30 e^{4}}+\frac{1}{e^{2}}-\frac{2}{e}\right)+\frac{3}{4} S_{3}\left(\frac{-1}{e^{2}}+\frac{3}{e}\right)+\frac{11}{3} S_{2}\left(\frac{1}{6 e^{2}}-\frac{1}{e}\right)+\frac{S_{1}}{e}\right] 5 n \\
& +\left(5 S_{4}-\frac{15}{2} S_{3}+\frac{55}{3} S_{2}-10 S_{1}\right) n .
\end{aligned}
$$

For a fixed $a, b, c, d$ and $e . g(a, b, c, d, e, n)$ is a function of $n$ and we denote it by $g(n)$.

We slice the five-dimensional simplex into the hyperplanes, $w=1, w=$ $2, \ldots, w=e-\beta-1$ and $w=e-\beta$. It is obvious there are no positive integral solutions on level $w=e-\beta$. At level $w=e-\beta-2$, the defining inequality of the simplex becomes $\frac{x}{\frac{a}{e}(\beta+2)}+\frac{y}{\frac{b}{e}(\beta+2)}+\frac{z}{\frac{c}{e}(\beta+2)}+\frac{v}{\frac{d}{e}(\beta+2)} \leq 1-$ note that $\frac{d}{e}(\beta+2)>3$ if $\beta=\frac{e}{d}$, and hence we can use GLY sharp upper estimate to bound the number of positive integral points at this level and for the levels lower than this level. At level $w=e-\beta-1$, the defining inequality of the simplex becomes $\frac{x}{\frac{a}{e}(\beta+1)}+\frac{y}{\frac{b}{e}(\beta+1)}+\frac{z}{\frac{c}{e}(\beta+1)}+\frac{v}{\frac{d}{e}(\beta+1)} \leq 1$, We divide our 
proof in two cases depending on whether the level $w=e-\beta-1$ tetrahedron

$$
\frac{x}{\frac{a}{e}(\beta+1)}+\frac{y}{\frac{b}{e}(\beta+1)}+\frac{z}{\frac{c}{e}(\beta+1)}+\frac{v}{\frac{d}{e}(\beta+1)} \leq 1
$$

has positive integral solutions.

Case (A). $\frac{a}{e}(1+\beta)<4$ or $\frac{b}{e}(1+\beta) \leq 3$ or $\frac{c}{e}(1+\beta) \leq 2$ or $\frac{d}{e}(1+\beta) \leq 1$.

In this case, there are no positive integral solutions at level $w=$ $e-\beta-1$.

Case (B). $\left.\frac{a}{e}(1+\beta) \geq 4, \frac{b}{e}(1+\beta)>3, \frac{c}{e}(1+\beta)\right)>2$, and $\frac{d}{e}(1+\beta)>1$.

In this case, (2.2) may have positive integral solutions.

Case (A).

Case (A1). $\beta=\frac{e}{d}$.

$\beta=\frac{e}{d}$ implies $\frac{d}{e}(\beta+2)>3$ - hence we can use the GLY sharp upper estimate to bound the number of positive integral points at this level and for the levels lower than this level. Without loss of generality, we can assume there exist positive integral solutions at level $w=e-\beta-2$ since the GLY upper estimate is positive at this level. Let $\Delta_{10}=$ RHS of $(2.1)-g(e-\beta-$ 2 ), and $A=\frac{a}{e}, B=\frac{b}{e}, C=\frac{c}{e}, D=\frac{d}{e}$ and $\beta=\frac{1}{D}$ and substitute into $\Delta_{10}$, then we have $A \geq B \geq C \geq D \geq 1$, and $e>4$. Let $\Delta_{11}=72 D^{4} \Delta_{10}$. Now all we have to do is partial differentiate to ensure that $\Delta_{11}$ is positive throughout the domain considered. If the level $w=e-\beta-2$ has positive integral solutions then $\left.\frac{a}{e}(2+\beta) \geq 4, \frac{b}{e}(2+\beta)>3, \frac{c}{e}(2+\beta)\right)>2$ and $\frac{d}{e}(2+\beta)>1$. We get $A \geq \frac{4 D}{2 D+1}, B>\frac{3 D}{2 D+1}, C \geq 1$, and $D \geq 1$. We find that $\Delta_{11}$ is an increasing function with respect to $A, B, C$ and $D$ for $A \geq \frac{4 D}{2 D+1}, B>\frac{3 D}{2 D+1}, C \geq 1$ and $D \geq 1$, and $e>4$. Evaluated at the minimum $A=\frac{4 D}{2 D+1}, B=\frac{3 D}{2 D+1}$, $C=1$ and $D=1, \Delta_{11}>0$, so we can conclude that $\Delta_{10}>0$ for this subcase.

$$
\begin{aligned}
\Delta_{10}= & \frac{1}{72 e^{4}}\left(-180 e^{3} a \beta^{2}-72 a d e^{4}+588 b c e^{4}-368 a c e^{5}\right. \\
& +135 a b c e^{3}+135 a b d e^{3}+135 a c d e^{3}+135 b c d e^{3} \\
& -360 e^{3} a-360 e^{3} b-360 e^{3} c-220 e^{3} a b \\
& -220 e^{3} a c-220 e^{3} b c-72 e^{4}+360 a b c d-120 a b c d e^{3}-72 b d e^{4} \\
& +1320 e^{2} a b+1320 e^{2} a c+1320 e^{2} b c-540 a b c e-540 a b d e \\
& -540 a c d e-540 b c d e+12 a b c d e-540 e^{3} a \beta-180 e^{3} b \beta^{2}-540 e^{3} b \beta \\
& -180 e^{3} c \beta^{2}-540 e^{3} c \beta-198 a b c e^{4}-198 a b d e^{4}-198 a c d e^{4} \\
& -198 b c d e^{4}+63 a b c e^{5}+63 a b d e^{5}+63 a c d e^{5}+63 b c d e^{5}+1980 e^{2} a c \beta^{2} \\
& +2860 e^{2} a c \beta+1980 e^{2} b c \beta^{2}+2860 e^{2} b c \beta+440 e^{2} a b \beta^{3}+1980 e^{2} a b \beta^{2}
\end{aligned}
$$


$+2860 e^{2} a b \beta+72 a d e^{5}-368 b c e^{5}-368 a b e^{5}-72 c d e^{4}-1080 e^{6} \beta$

$-360 \beta^{4} e^{4}-360 \beta^{3} e^{4}+1440 e^{5} \beta^{3}-2160 e^{6} \beta^{2}+1440 e^{7} \beta$

$+72 c d e^{5}+1080 e^{5} \beta^{2}+360 \beta^{2} e^{4}-720 e^{5} \beta-72 d e^{5}$

$+108 c e^{5}+108 b e^{5}+108 a e^{5}+432 \beta e^{4}+72 d e^{4}-108 c e^{4}$

$-108 b e^{4}-360 e^{5}+360 e^{7}+360 e^{6}-360 e^{8}-108 a e^{4}+72 b d e^{5}$

$+588 a c e^{4}+588 a b e^{4}+108 a b c d e^{4}+2160 a b c d \beta^{2}+1560 a b c d \beta^{3}$

$+540 a b c d \beta^{4}+1428 a b c d \beta+72 a b c d \beta^{5}-810 a b c e \beta^{3}-1755 a b c e \beta^{2}$

$-1620 a b c e \beta+440 e^{2} a c \beta^{3}+440 e^{2} b c \beta^{3}-810 a b d e \beta^{3}-1755 a b d e \beta^{2}$

$-1620 a b d e \beta-810 a c d e \beta^{3}-1755 a c d e \beta^{2}-1620 a c d e \beta-810 b c d e \beta^{3}$

$-1755 b c d e \beta^{2}-1620 b c d e \beta-135 a b c e \beta^{4}-135 a b d e \beta^{4}$

-135 acde $\left.\beta^{4}-135 b c d e \beta^{4}\right)$,

$\Delta_{11}=-360-360 D-72 A e^{2} D^{5}-108 C e D^{4}-540 C D^{3}+72 C e^{3} D^{5}$

$-180 B D^{2}-72 C e^{2} D^{5}-72 B e^{2} D^{5}-540 B D^{3}-360 C D^{4}-180 C D^{2}$

$+108 A e^{2} D^{4}-360 B D^{4}-360 A D^{4}-540 A D^{3}-108 B e D^{4}$

$+108 C e^{2} D^{4}+72 A e^{3} D^{5}+108 B e^{2} D^{4}+72 B e^{3} D^{5}-360 D^{4} e^{4}$

$-180 A D^{2}-108 A e D^{4}+1440 D e+135 B e^{2} C D^{5}-63 A B C$

$-300 A C D^{4}+63 A e^{4} B D^{5}-198 A e^{3} B D^{5}-368 A e^{3} B D^{4}$

$+135 A e^{2} B D^{5}+588 A e^{2} B D^{4}-220 A e B D^{4}-540 A B D^{5}$

$-300 A B D^{4}+1105 A B D^{3}+1170 A B D^{2}+305 A B D+63 A e^{4} C D^{5}$

$-198 A e^{3} C D^{5}-368 A e^{3} C D^{4}+135 A e^{2} C D^{5}+588 A e^{2} C D^{4}$

$-220 A e C D^{4}-540 A C D^{5}+1105 A C D^{3}+1170 A C D^{2}+305 A C D$

$+1170 B C D^{2}+1105 B C D^{3}+305 B C D$

$+588 B e^{2} C D^{4}-220 B e C D^{4}-300 B C D^{4}-540 B C D^{5}$

$-198 B e^{3} C D^{5}+63 B e^{4} C D^{5}-368 B e^{3} C D^{4}+360 D^{4} e^{3}-72 D^{5} e^{2}$

$+360 D^{4} e^{2}+1440 D^{3} e^{3}-720 D^{3} e-360 D^{4} e+1080 D^{2} e-1080 D^{3} e^{2}$

$+432 D^{3}+360 D^{2}-72 D^{4}-2160 D^{2} e^{2}+72 D^{5} e+108 A e^{4} B C D^{5}$

$+63 A e^{4} B C D^{4}-120 A e^{3} B C D^{5}-198 A e^{3} B C D^{4}+135 A e^{2} B C D^{4}$

$+12 A e B C D^{5}+360 A B C D^{5}+888 A B C D^{4}+540 A B C D^{3}$

$-195 A B C D^{2}-270 A B C D$.

$\left.\Delta_{11}\right|_{A=\frac{4 D}{2 D+1}, B=\frac{3 D}{2 D+1}, C=1, D=1}$

$=4460+\frac{1084}{3} e-\frac{1378}{3} e^{3}-e^{2}+99 e^{4} \geq 0$ for $e \geq 4$ 
Therefore we have $\Delta_{11} \geq 0$ on the region considered.

Case (A2). $\beta=\frac{e}{c}$. If $\frac{d}{e}(\beta+2) \geq 3$, then the GLY sharp upper estimate or GLY rough upper estimate can be used; if $1<\frac{d}{e}(\beta+2)<3$, we must use GLY rough upper estimate. Let

$$
\begin{aligned}
\Delta_{12}= & \operatorname{RHS} \text { of }(2.1)-g(e-\beta-3) \\
& -5(A(\beta+2)-1)(B(\beta+2)-1)(C(\beta+2)-1)(D(\beta+2)-1) .
\end{aligned}
$$

We use GLY rough upper estimate at level $w=e-\beta-2$, and without loss of generality, we can assume there exist positive integral solutions at level $w=$ $e-\beta-2$ since the GLY rough upper estimate is positive at this level. From level $w=1$ to level $w=e-\beta-3, d\left(1-\frac{w}{e}\right) \geq 3$ and so the GLY sharp upper estimate is used. If the level $w=e-\beta-2$ has positive integral solutions then we have $\frac{a}{e}(2+\beta) \geq 4, \frac{b}{e}(2+\beta)>3, \frac{c}{e}(2+\beta)>2$ and $\frac{d}{e}(2+\beta)>1$. Hence $A \geq \frac{4 C}{2 C+1}, B>\frac{3 C}{2 C+1}, C \geq 1$ and $D \geq 1$. Let $A=\frac{a}{e}, B=\frac{b}{e}, C=\frac{c}{e}$, $D=\frac{d}{e}$ and $\beta=\frac{1}{C}$ and substitute into $\Delta_{12}$.

Let $\Delta_{13}=72 C^{4} \Delta_{12}$. We find that $\Delta_{13}$ is an increasing function with respect to $A, B, C$ and $D$ for $A \geq \frac{4 C}{2 C+1}, B>\frac{3 C}{2 C+1}, C \geq 1$ and $D \geq 1$ and $e>4$. Evaluated at the minimum $A=\frac{4 C}{2 C+1}, B=\frac{3 C}{2 C+1}, C=1$, and $D=1$, $\Delta_{13}>0$, so we can conclude that $\Delta_{12}>0$ for $e \geq 4.194$, and we consider $4<e<4.194$ in Case (A5).

$$
\begin{aligned}
\Delta_{12}= & \frac{1}{72 e^{4}}\left(6120 a b c d-120 a b c d e^{3}+135 a b c e^{3}+135 a b d e^{3}+135 a c d e^{3}\right. \\
& +135 b c d e^{3}-360 e^{4} A \beta^{2} B-1440 e^{4} A B+6600 e^{2} a b+6600 e^{2} a c \\
& +6600 e^{2} b c+12 a b c d e-4860 a b c e-4860 a b d e-4860 a c d e-4860 b c d e \\
& -180 e^{3} a \beta^{2}+72 d e^{4}-108 c e^{4}+72 b d e^{5}-1080 e^{6} \beta+1440 e^{5} \beta^{3} \\
& -2160 e^{6} \beta^{2}+1440 e^{7} \beta+1080 e^{5} \beta^{2}+360 \beta^{2} e^{4}-720 e^{5} \beta-72 d e^{5} \\
& +108 c e^{5}+108 b e^{5}+108 a e^{5}-360 \beta^{4} e^{4}-360 \beta^{3} e^{4}+432 \beta e^{4} \\
& -108 b e^{4}-108 a e^{4}-198 a b c e^{4}-198 a b d e^{4}-198 a c d e^{4}-198 b c d e^{4} \\
& +63 a b c e^{5}+63 a b d e^{5}+63 a c d e^{5}+63 b c d e^{5}-360 e^{4} B \beta^{2} C \\
& -1440 e^{4} A \beta D-1440 e^{4} B \beta C-360 e^{4} B \beta^{2} D-360 e^{5} \\
& +360 e^{7}+360 e^{6}-360 e^{8}+108 a b c d e^{4}+12948 a b c d \beta+4440 a b c d \beta^{3} \\
& +10800 a b c d \beta^{2}+900 a b c d \beta^{4}+72 a b c d \beta^{5}+720 e^{4} D+720 e^{4} A \\
& +720 e^{4} B+720 e^{4} C-1440 e^{4} A \beta B-1440 e^{4} A \beta C-360 e^{4} A \beta^{2} D \\
& +2880 e^{4} A C D-1440 e^{4} B \beta D+2880 e^{4} A B D-360 e^{4} C \beta^{2} D \\
& -1440 e^{4} C \beta D+2880 e^{4} B C D+2880 e^{4} A B C-360 e^{4} A \beta^{2} C \\
&
\end{aligned}
$$




$$
\begin{aligned}
& +360 e^{4} A \beta^{3} B C+2160 e^{4} A \beta^{2} B C+4320 e^{4} A \beta B C+360 e^{4} A \beta^{3} B D \\
& +360 e^{4} A \beta^{3} C D+360 e^{4} B \beta^{3} C D+2160 e^{4} A \beta^{2} B D+4320 e^{4} A \beta B D \\
& +2160 e^{4} A \beta^{2} C D+4320 e^{4} A \beta C D-5760 e^{4} A B C D+2160 e^{4} B \beta^{2} C D \\
& +4320 e^{4} B \beta C D-360 e^{4} A \beta^{4} B C D-2880 e^{4} A \beta^{3} B C D \\
& -8640 e^{4} A \beta^{2} B C D-11520 e^{4} A \beta B C D-1350 a b c e \beta^{3}-4995 a b c e \beta^{2} \\
& -8100 a b c e \beta+3300 e^{2} a b \beta^{2}+8140 e^{2} a b \beta-1350 a b d e \beta^{3}-4995 a b d e \beta^{2} \\
& -8100 a b d e \beta-1350 a c d e \beta^{3}-4995 a c d e \beta^{2}-8100 a c d e \beta-1350 b c d e \beta^{3} \\
& -4995 b c d e \beta^{2}-8100 b c d e \beta-135 a b c e \beta^{4}-135 a b d e \beta^{4}-135 a c d e \beta^{4} \\
& -135 b c d e \beta^{4}+3300 e^{2} a c \beta^{2}+8140 e^{2} a c \beta+3300 e^{2} b c \beta^{2}+8140 e^{2} b c \beta \\
& +440 e^{2} a b \beta^{3}+440 e^{2} a c \beta^{3}+440 e^{2} b c \beta^{3}+588 b c e^{4}-220 e^{3} a b \\
& -220 e^{3} a c-220 e^{3} b c-180 e^{3} b \beta^{2}+588 a b e^{4}-1080 e^{3} a-1080 e^{3} b \\
& -1080 e^{3} c-900 e^{3} c \beta-432 e^{4}-368 a c e^{5}-368 b c e^{5}-72 b d e^{4}-72 c d e^{4} \\
& +72 c d e^{5}-368 a b e^{5}-1440 e^{4} B C-1440 e^{4} A D-1440 e^{4} B D \\
& -1440 e^{4} C D+360 e^{4} A \beta+360 e^{4} B \beta+360 e^{4} C \beta+360 e^{4} D \beta-72 a d e^{4} \\
& \left.-180 e^{3} c \beta^{2}+72 a d e^{5}-900 e^{3} a \beta-900 e^{3} b \beta+588 a c e^{4}-1440 e^{4} A C\right) \text {, } \\
& \Delta_{13}=-360+72 D e C^{4}+588 A e^{2} C^{5}+5160 A C^{5}+72 C^{5} e^{3} D \\
& -368 A e^{3} C^{5}-720 D C^{4}+5160 B C^{5}-108 B e C^{4}+6340 B C^{4} \\
& -1080 e^{2} C^{3}-360 C-450 A B C D+1440 C e-220 e A C^{5} \\
& -5220 A C^{4} D+1380 A B C^{4}-5220 B D C^{4}-1980 A C^{5} D \\
& -1980 B C^{5} D-1980 A B C^{5}-4275 A D C^{3}-1350 B D C^{2} \\
& +3865 A B C^{3}-1350 A D C^{2}-4275 B D C^{3}+1950 A B C^{2} \\
& +135 A e^{2} B C^{5}-198 A e^{3} B C^{5}+63 A e^{4} B C^{5}+360 e^{2} C^{4}-360 e^{4} C^{4} \\
& +360 e^{3} C^{4}-108 C^{5} e+108 C^{5} e^{2}-2160 e^{2} C^{2}+1440 e^{3} C^{3}-360 e C^{4} \\
& +1080 e C^{2}-720 e C^{3}+252 C^{3}+360 C^{2}-360 C^{5}-972 C^{4} \\
& -552 A B D C^{4}+360 A B C^{5} D-1275 A B D C^{2}-1620 A B D C^{3} \\
& -220 e A B C^{4}+588 A e^{2} B C^{4}-368 A e^{3} B C^{4} \\
& +72 A e^{3} D C^{4}+135 A e^{2} C^{5} D-198 A e^{3} C^{5} D+63 A e^{4} C^{5} D \\
& -72 A e^{2} D C^{4}+135 B e^{2} C^{5} D+72 B e^{3} D C^{4}-198 B e^{3} C^{5} D \\
& +63 B e^{4} C^{5} D-72 B e^{2} D C^{4}-120 A e^{3} B C^{5} D+135 A e^{2} B D C^{4} \\
& +12 A e B C^{5} D-198 A e^{3} B D C^{4}+63 A e^{4} B D C^{4}+108 A e^{4} B C^{5} D \\
& -368 B e^{3} C^{5}+2400 A C^{3}+260 A C^{2}+108 A e^{2} C^{4}-135 A C D \\
& -63 A B D-108 A e C^{4}+260 B C^{2}+108 B e^{2} C^{4}+588 B e^{2} C^{5}
\end{aligned}
$$




$$
\begin{aligned}
& -72 C^{5} e^{2} D-72 D e^{2} C^{4}+2400 B C^{3}-135 B C D-1440 C^{5} D \\
& +6340 A C^{4}+305 A B C-220 e B C^{5}, \\
\left.\Delta_{13}\right|_{A=} & \frac{4 C}{2 C+1}, B=\frac{3 C}{2 C+1}, C=1, D=1 \\
& =1760+\frac{1084}{3} e-\frac{1378}{3} e^{3}-e^{2}+99 e^{4}>0 \quad \text { for } e \geq 4.194
\end{aligned}
$$

Therefore, we have $\Delta_{12} \geq 0$ for $e \geq 4.194$.

Case (A3). $\beta=\frac{e}{b}$. Similar to Case(A2). Substitute $\beta=\frac{e}{b}$ into $\Delta_{12}$. Let $\Delta_{14}=72 B^{4} \Delta_{12}$. We find that $\Delta_{14}$ is an increasing function with respect to $A, B, C$ and $D$ for $A \geq \frac{4 B}{2 B+1}, B \geq 1, C \geq 1$ and $D \geq 1$ and $e>4$. Evaluated at the minimum $A=\frac{4 B}{2 B+1}, B=1, C=1$ and $D=1, \Delta_{14}>0$ for $e \geq 4.194$, so we can conclude that $\Delta_{12}>0$ for $e \geq 4.194$, and we consider $4<e<4.194$ in Case (A5).

$$
\begin{aligned}
\Delta_{14}= & -360+72 D e B^{4}+6340 C B^{4}+5160 B^{5} C+260 A B^{2}-108 A e B^{4} \\
& -1440 B^{5} D-220 e A B^{5}+260 C B^{2}-368 A e^{3} B^{5}+2400 A B^{3} \\
& +6340 A B^{4}-450 A B C D+1440 B e-368 B^{5} e^{3} C+108 C e^{2} B^{4} \\
& -220 e B^{5} C+108 A e^{2} B^{4}-5220 A D B^{4}-5220 C D B^{4} \\
& +1380 A C B^{4}-1980 A B^{5} D-1980 B^{5} C D-1980 A B^{5} C \\
& +3865 A C B^{3}-1350 A D B^{2}-1350 C D B^{2}-4275 C D B^{3} \\
& +1950 A C B^{2}-4275 A B^{3} D+135 A e^{2} B^{5} C-198 A e^{3} B^{5} C \\
& +63 A e^{4} B^{5} C-220 e A C B^{4}-368 A e^{3} C B^{4}+588 A e^{2} C B^{4} \\
& -198 B^{5} e^{3} C D+72 C e^{3} D B^{4}+135 A e^{2} B^{5} D+63 A e^{4} B^{5} D \\
& -72 A e^{2} D B^{4}+72 A e^{3} D B^{4}+135 B^{5} e^{2} C D+63 B^{5} e^{4} C D \\
& +360 e^{2} B^{4}-1080 e^{2} B^{3}-360 e B^{4}-720 e B^{3}+360 B^{2}+1440 e^{3} B^{3} \\
& -2160 e^{2} B^{2}+1080 e B^{2}+108 B^{5} e^{2}+360 e^{3} B^{4}-360 B^{5}-972 B^{4} \\
& +252 B^{3}-1275 A C D B^{2}-1620 A C D B^{3}-72 C e^{2} D B^{4} \\
& -198 A e^{3} B^{5} D-120 A e^{3} B^{5} C D+135 A e^{2} C D B^{4}+12 A e B^{5} C D \\
& -198 A e^{3} C D B^{4}+63 A e^{4} C D B^{4}+108 A e^{4} B^{5} C D-63 A C D \\
& -72 D e^{2} B^{4}-720 D B^{4}-135 A B D-108 C e B^{4}+2400 C B^{3} \\
& +360 A B^{5} C D-360 e^{4} B^{4}+72 B^{5} e^{3} D+588 B^{5} e^{2} C-135 B C D \\
& -72 B^{5} e^{2} D+5160 A B^{5}+588 A e^{2} B^{5}-360 B+305 A B C \\
& -108 B^{5} e-552 A C D B^{4},
\end{aligned}
$$




$$
\begin{aligned}
& \left.\Delta_{14}\right|_{A=\frac{4 B}{2 B+1}, B=1, C=1, D=1} \\
& \quad=1760+\frac{1084}{3} e-\frac{1378}{3} e^{3}-e^{2}+99 e^{4}>0 \quad \text { for } e \geq 4.194
\end{aligned}
$$

Therefore, we have $\Delta_{12} \geq 0$ for $e \geq 4.194$.

Case (A4). $\beta=\frac{e}{a}$. Similar to Case (A2). Substitute $\beta=\frac{e}{a}$ into $\Delta_{12}$. Let $\Delta_{15}=72 A^{4} \Delta_{12}$. We find that $\Delta_{15}$ is an increasing function with respect to $A, B, C$ and $D$ for $A \geq 1.5, B \geq \frac{3 A}{2 A+1}, C \geq 1$ and $D \geq 1$ and $e>4$. Evaluated at the minimum $A=1.5, B=\frac{3 A}{2 A+1}, C=1$ and $D=1, \Delta_{15}>0$, for $e \geq 4.896$, so we can conclude that $\Delta_{12}>0$ for $e \geq 4.896$, and we consider $4<e<4.896$ at Case (A5).

$$
\begin{aligned}
\Delta_{15}= & -360-108 B e A^{4}-220 e A^{5} B-450 A B C D+1440 A e+1080 e A^{2} \\
& +5160 A^{5} B-1440 A^{5} D+5160 A^{5} C+588 A^{5} e^{2} B+260 B A^{2} \\
& -368 A^{5} e^{3} B-108 C e A^{4}+108 C e^{2} A^{4}-220 e A^{5} C+260 C A^{2} \\
& -368 A^{5} e^{3} C+588 A^{5} e^{2} C+72 D e A^{4}-72 D e^{2} A^{4}-72 A^{5} e^{2} D \\
& +72 A^{5} e^{3} D+2400 A^{3} C+63 A^{5} e^{4} B C-972 A^{4}-1080 e^{2} A^{3} \\
& -2160 e^{2} A^{2}-360 e^{4} A^{4}+360 e^{2} A^{4}+360 e^{3} A^{4}-360 e A^{4} \\
& +108 A^{5} e^{2}-108 A^{5} e-720 e A^{3}+252 A^{3}+360 A^{2}-360 A^{5} \\
& +1440 e^{3} A^{3}+1950 B C A^{2}+1380 B C A^{4}-5220 B D A^{4}-5220 C D A^{4} \\
& -1980 A^{5} C D-1980 A^{5} B D-1980 A^{5} B C+3865 B C A^{3}-552 B C D A^{4} \\
& +360 A^{5} B C D-4275 B D A^{3}-1350 C D A^{2}-4275 C D A^{3}-1350 A^{2} B D \\
& +135 A^{5} e^{2} B C-198 A^{5} e^{3} B C+588 B e^{2} C A^{4}-220 e B C A^{4} \\
& -368 B e^{3} C A^{4}+72 B e^{3} D A^{4}-198 A^{5} e^{3} B D+63 A^{5} e^{4} B D \\
& -72 B e^{2} D A^{4}+135 A^{5} e^{2} B D-198 A^{5} e^{3} C D+63 A^{5} e^{4} C D-72 C e^{2} D A^{4} \\
& +72 C e^{3} D A^{4}+135 A^{5} e^{2} C D-1275 B C D A^{2}-1620 A^{3} B C D \\
& -120 A^{5} e^{3} B C D+135 B e^{2} C D A^{4}+12 A^{5} e B C D-198 B e^{3} C D A^{4} \\
& +63 B e^{4} C D A^{4}+108 A^{5} e^{4} B C D-720 D A^{4}-135 A C D-135 A B D \\
& +6340 C A^{4}-360 A+6340 B A^{4}-63 B C D+108 B e^{2} A^{4}+305 A B C \\
& +2400 A^{3} B
\end{aligned}
$$

$$
\begin{aligned}
\left.\Delta_{15}\right|_{A=1.5, B=\frac{3 A}{2 A+1}, C=1, D=1}= & -7069.781 e^{3}-5980.5 e+23593.5 \\
& +10551.516 e^{2}+1013.766 e^{4}>0 \text { for } e \geq 4.896 .
\end{aligned}
$$


Case (A5). $e \in(4,4.9)$. For $e \in(4,4.9)$, consider the following two subcases:

(a) The level $w=e-\beta-2=2$ has no positive integral solutions: $P_{4}(2)=0$.

(b) The level $w=e-\beta-2=2$ has positive integral solutions: $P_{4}(2)>0$.

In Case (A5) the RHS of (2.1) becomes

$$
\begin{aligned}
\mu- & \left.\left(5 v^{4}-25 v^{3}+40 v^{2}-19 v-1\right)\right|_{v=5} \\
\quad= & (a-1)(b-1)(c-1)(d-1)(e-1)-904 .
\end{aligned}
$$

For subcase (a), the proof is almost exactly the same as the proof presented in Case IV. Because $P_{5}>0$, the set of positive integral solutions includes the point $(1,1,1,1,1)$, and let $\frac{1}{a}+\frac{1}{b}+\frac{1}{c}+\frac{1}{d} \leq 1-\frac{1}{e} \triangleq \alpha, \alpha \in$ $\left(\frac{3}{4}, \frac{39}{49}\right)$, since $e \in(4,4.9)$. Let $A=a \alpha, B=b \alpha, C=c \alpha$ and $D=d \alpha$; then, the following restrictions apply:

$$
A \geq 4, B \geq 3, C \geq 2, D \geq \frac{\alpha}{1-\alpha},
$$

because $D=d \alpha \geq e \alpha=\frac{\alpha}{1-\alpha}$. With $\alpha \in\left(\frac{3}{4}, \frac{39}{49}\right), \frac{\alpha}{1-\alpha} \in(3,3.9)$.

$$
\begin{aligned}
5 ! P_{5} \leq & 5[(A-1)(B-1)(C-1)(D-1) \\
& \left.-(D-1)^{4}+D(D-1)(D-2)(D-3)\right] .
\end{aligned}
$$

Let $\Delta_{16}=$ RHS of (2.3) - RHS of (2.4), and substitute $A=\frac{a}{e}, B=\frac{b}{e}, C=$ $\frac{c}{e}$ and $D=\frac{d}{e}$ into $\Delta_{16}$

$$
\begin{aligned}
\Delta_{16}= & -905+5 A+5 B+5 C+15 D+e+A e^{3} B C+A e^{3} B D+A e^{3} C D \\
& +B e^{3} C D-A e^{4} B C-A e^{4} B D-A e^{4} C D-B e^{4} C D-5 A B C D \\
& +A e+B e+C e+D e-A e^{2}-B e^{2}-C e^{2}-D e^{2}-5 A B-5 A C \\
& -5 B C-5 A D-5 B D-5 C D-25 D^{2}-A e^{2} B-A e^{2} C-B e^{2} C \\
& -A e^{2} D-B e^{2} D-C e^{2} D+A e^{3} B+A e^{3} C+A e^{3} D+B e^{3} C+B e^{3} D \\
& +C e^{3} D+5 A B C+5 A B D+5 A C D+5 B C D-A e^{4} B C D \\
& +A e^{5} B C D+10 D^{3},
\end{aligned}
$$

$$
\begin{aligned}
\left.\Delta_{16}\right|_{A=4, B=3, C=3, D=3} & =-945+14 e-76 e^{2}+198 e^{3}-243 e^{4}+108 e^{5} \\
& >0 \text { for } e>4 .
\end{aligned}
$$

For subcase (b), $P_{4}(2)>0$, which implies that $(1,1,1,1,2)$ is the smallest positive integer solution to the level $w=2$. Then, we have $\frac{1}{a}+\frac{1}{b}+\frac{1}{c}+\frac{1}{d} \leq$ 
$1-\frac{2}{e} \triangleq \alpha, \alpha \in\left(\frac{1}{2}, \frac{29}{49}\right)$, since $e \in(4,4.9)$. Let $A=a \alpha, B=b \alpha, C=c \alpha$ and $D=d \alpha$, and then we know $A \geq 4, B \geq 3, C \geq 2, D>1, D=d \alpha \geq e \alpha=$ $\frac{2 \alpha}{1-\alpha}$. With $\alpha \in\left(\frac{1}{2}, \frac{29}{49}\right), \frac{2 \alpha}{1-\alpha} \in(2,2.9)$, we obtain the following:

$$
\begin{aligned}
5 ! P_{5}= & 5 !\left(P_{4}(1)+P_{4}(2)\right) \\
\leq & 5\left[\left(A\left(\frac{1-\alpha}{2 \alpha}+1\right)-1\right)\left(B\left(\frac{1-\alpha}{2 \alpha}+1\right)-1\right)\right. \\
& \cdot\left(C\left(\frac{1-\alpha}{2 \alpha}+1\right)-1\right)\left(D\left(\frac{1-\alpha}{2 \alpha}+1\right)-1\right) \\
& -\left(D\left(\frac{1-\alpha}{2 \alpha}+1\right)-1\right)+D\left(\frac{1-\alpha}{2 \alpha}+1\right) \\
& +\left(D\left(\frac{1-\alpha}{2 \alpha}+1\right)-1\right)\left(D\left(\frac{1-\alpha}{2 \alpha}+1\right)-2\right) \\
& \cdot\left(D\left(\frac{1-\alpha}{2 \alpha}+1\right)-3\right) \\
& +(A-1)(B-1)(C-1)(D-1)-(D-1)^{4} \\
& +D(D-1)(D-2)(D-3)] .
\end{aligned}
$$

Let $\Delta_{17}=$ RHS of (2.3) - RHS of (2.5) and by substituting $a=\frac{A}{\alpha}, b=$ $\frac{B}{\alpha}, c=\frac{C}{\alpha}, d=\frac{D}{\alpha}, e=\frac{2}{1-\alpha}$ into $\Delta_{17}$,

$$
\begin{aligned}
16 \alpha^{4} \Delta_{17}= & \alpha^{4}\left(-500 D^{2}-16 B e^{2}-85 A B C D+16 B e^{3} D-16 C e^{2}\right. \\
& -16 D e^{2}-16 A e^{2} B-16 A e^{2} C-16 B e^{2} C-16 A e^{2} D-16 B e^{2} D \\
& -16 C e^{2} D+16 A e^{3} B+16 A e^{3} C+16 A e^{3} D+16 B e^{3} C-100 A C \\
& +16 C e^{3} D+90 A B C+90 A B D+90 A C D+90 B C D \\
& +120 A+120 B+120 C+360 D+180 D^{3}-16 A e^{4} B C D \\
& +16 A e^{5} B C D+16 A e^{3} B D-100 A D-100 B C+16 A e^{3} B C \\
& +16 B e+16 e-14480+16 A e-100 A B+16 C e+16 D e-16 A e^{2} \\
& -100 B D-100 C D-16 A e^{4} B D+16 A e^{3} C D+16 B e^{3} C D \\
& \left.-16 A e^{4} B C-16 A e^{4} C D-16 B e^{4} C D\right) \\
& +\alpha^{3}\left(-40 C D+40 B+60 D^{3}+30 B C D+40 A-40 B D+40 C\right. \\
& -20 A B C D+30 A B C-200 D^{2}-40 A C+30 A B D-40 A D \\
& -40 B C+120 D+30 A C D-40 A B)
\end{aligned}
$$




$$
\begin{aligned}
& +\alpha^{2}\left(30 B C D-20 A B-20 A C-20 B D+60 D^{3}-20 A D\right. \\
& +30 A B C+30 A C D-100 D^{2}-20 C D-30 A B C D \\
& -20 B C+30 A B D)+\alpha\left(10 B C D+20 D^{3}+10 A B C\right. \\
& +10 A B D+10 A C D-20 A B C D)-5 A B C D,
\end{aligned}
$$

$\left.16 \alpha^{4} \Delta_{17}\right|_{A=4, B=3, C=2, D=2}$

$$
\begin{aligned}
= & -240+192 e \alpha^{4}-880 e^{2} \alpha^{4}-40 \alpha+40 \alpha^{2}+768 e^{5} \alpha^{4} \\
& -14880 \alpha^{4}-1984 e^{4} \alpha^{4}+1920 e^{3} \alpha^{4}-160 \alpha^{3} \\
> & 0 \quad \text { for } \alpha \in\left(\frac{1}{2}, \frac{29}{49}\right) e>4 .
\end{aligned}
$$

Case $\left.(B) . \frac{a}{e}(1+\beta) \geq 4, \quad \frac{b}{e}(1+\beta)>3, \quad \frac{c}{e}(1+\beta)\right)>2$ and $\frac{d}{e}(1+\beta)>1$, where $0<\beta<1$.

Case (B1). $\beta=\frac{e}{d}$. If there are no positive lattice points on level $w=e-$ $\beta-1$, the proof is the same as Case (A1), and so we can assume there exist positive integral solutions at level $w=e-\beta-1$. $\beta=\frac{e}{d}$ implies $\frac{d}{e}(\beta+1)>$ 2 ; hence we can use GLY sharp upper estimate to bound the number of positive integral points at this level and for the levels lower than this level. Let $\Delta_{18}=$ RHS of $(2.1)-g(e-\beta-1)$. It suffices to prove that $\Delta_{18}>0$. Let $A=\frac{a}{e}, B=\frac{b}{e}, C=\frac{c}{e}, D=\frac{d}{e}$ and $\beta=\frac{1}{D}$ - then substitute into $\Delta_{18}$. Now all we have to do is partial differentiate to ensure that $\Delta_{18}$ is positive throughout the domain considered. From $A(1+\beta) \geq 4, B(1+\beta)>3, C(1+\beta)>2$ and $D(1+\beta)>1$ and $\beta=\frac{1}{D}$, we obtain $A \geq \frac{4 D}{D+1}, B>\frac{3 D}{D+1}, C>\frac{2 D}{D+1}$ and $D>$ 1. Let $\Delta_{19}=72 D^{4} \Delta_{18}$. We find that $\Delta_{19}$ is an increasing function with respect to $A, B, C$ and $D$ for $A \geq \frac{4 D}{D+1}, B>\frac{3 D}{D+1}, C>\frac{2 D}{D+1}$ and $D>1$, and $e>4$. Evaluated at the minimum $A=\frac{4 D}{D+1}, B=\frac{3 D}{D+1}, C=1$ and $D=1$, $\Delta_{19}>0$, so we can conclude that $\Delta_{18}>0$ for this subcase.

$$
\begin{aligned}
\Delta_{18}= & \frac{1}{72 e^{4}}\left(-72 c d e^{4}+135 a b c e^{3}+135 a b d e^{3}+135 a c d e^{3}+135 b c d e^{3}\right. \\
& -180 e^{3} b \beta^{2}-180 e^{3} c \beta-180 e^{3} c \beta^{2}-72 e^{4}-220 e^{3} a c-220 e^{3} b c \\
& +72 a d e^{5}-198 a b d e^{4}-198 a c d e^{4}-198 b c d e^{4}+63 a b c e^{5}+63 a b d e^{5} \\
& +63 a c d e^{5}+63 b c d e^{5}+440 e^{2} a c \beta^{3}+440 e^{2} b c \beta^{3}+220 e^{2} a b \beta \\
& +220 e^{2} a c \beta+220 e^{2} b c \beta+588 b c e^{4}+588 a b e^{4}-72 b d e^{4} \\
& +108 b e^{5}+108 a e^{5}-360 \beta^{4} e^{4}-1080 e^{6} \beta+1440 e^{5} \beta^{3} \\
& -2160 e^{6} \beta^{2}+1440 e^{7} \beta+1080 e^{5} \beta^{2}+360 \beta^{2} e^{4}
\end{aligned}
$$




$$
\begin{aligned}
& -720 e^{5} \beta-72 d e^{5}-360 \beta^{3} e^{4}+108 c e^{5}+432 \beta e^{4} \\
& -108 b e^{4}-108 a e^{4}+72 d e^{4}-108 c e^{4}-360 e^{5}+360 e^{7}+360 e^{6} \\
& -360 e^{8}+108 a b c d e^{4}-368 a b e^{5}-368 a c e^{5}+72 b d e^{5}-180 e^{3} a \beta \\
& +120 a b c d \beta^{3}+180 a b c d \beta^{4}+72 a b c d \beta^{5}+660 e^{2} a b \beta^{2} \\
& +660 e^{2} a c \beta^{2}+660 e^{2} b c \beta^{2}+440 e^{2} a b \beta^{3}-135 a b c e \beta^{4} \\
& -135 a b c e \beta^{2}-135 a b d e \beta^{4}-135 a b d e \beta^{2}-135 a c d e \beta^{4} \\
& -135 a c d e \beta^{2}-135 b c d e \beta^{4}-135 b c d e \beta^{2}-180 e^{3} b \beta \\
& -270 a b c e \beta^{3}-270 a b d e \beta^{3}-270 a c d e \beta^{3}-270 b c d e \beta^{3} \\
& \left.-12 a b c d \beta+12 a b c d e-72 a d e^{4}+588 a c e^{4}\right), \\
& \Delta_{19}=-360-360 D-108 C e D^{4}-180 B D^{3}-180 C D^{3}-180 A D^{2} \\
& +72 A e^{3} D^{5}+360 e^{2} D^{4}-120 A e^{3} B C D^{5}-72 D^{5} e^{2}-72 B e^{2} D^{5} \\
& -72 D^{4}+432 D^{3}+305 A C D+305 B C D+390 A B D^{2}+85 A B D^{3} \\
& -220 e A B D^{4}+360 e^{3} D^{4}+360 D^{2}+72 D^{5} e-360 e D^{4}+1440 e^{3} D^{3} \\
& -2160 e^{2} D^{2}-720 e D^{3}+1080 e D^{2}-12 A B C D^{4}-198 A e^{3} C D^{5} \\
& +588 e^{2} A C D^{4}+63 A e^{4} C D^{5}-368 A e^{3} C D^{4}+135 A e^{2} C D^{5} \\
& -220 e A C D^{4}+85 A C D^{3}+390 A C D^{2}+85 B C D^{3} \\
& -90 A B C D+12 A e B C D^{5}-15 A B C D^{2}-198 A e^{3} B C D^{4} \\
& +108 A e^{4} B C D^{5}+63 A e^{4} B C D^{4}+135 A e^{2} B C D^{4}+1440 D e \\
& -1080 e^{2} D^{3}-360 e^{4} D^{4}-198 B e^{3} C D^{5}+588 e^{2} B C D^{4}+63 B e^{4} C D^{5} \\
& -368 B e^{3} C D^{4}+135 B e^{2} C D^{5}-220 e B C D^{4}+390 B C D^{2}-63 A B C \\
& +305 A B D-198 A e^{3} B D^{5}+588 e^{2} A B D^{4}+63 A e^{4} B D^{5} \\
& -368 A e^{3} B D^{4}+135 A e^{2} B D^{5}-180 C D^{2}+108 A e^{2} D^{4}-72 C e^{2} D^{5} \\
& -72 A e^{2} D^{5}-108 A e D^{4}+72 C e^{3} D^{5}-180 A D^{3}-108 B e D^{4} \\
& +108 C e^{2} D^{4}-180 B D^{2}+72 B e^{3} D^{5}+108 B e^{2} D^{4}, \\
& \left.\Delta_{19}\right|_{A=\frac{4 D}{D+1}, B=\frac{3 D}{D+1}, C=1, D=1} \\
& =2910-368 e-2509 e^{3}+\frac{4629}{2} e^{2}+\frac{1125}{2} e^{4} \geq 0 \text { for } e \geq 4 .
\end{aligned}
$$

Therefore, we have $\Delta_{18} \geq 0$ on the region considered.

Case (B2). $\beta=\frac{e}{c}$. If there are no positive lattice points at level $w=e-\beta-$ 1 , then the proof is the same as Case (A2), and so we can assume there exist positive integral solutions at level $w=e-\beta-1 \cdot \frac{d}{e}(\beta+2)>2$ - hence we can use the GLY sharp upper estimate to bound the number of positive integral points at level $w=e-\beta-2$ and for the levels lower than level 
$w=e-\beta-2$. We use Theorem 1.3 to bound the number of positive integral points at level $w=e-\beta-1$. Let

$$
\begin{aligned}
\Delta_{20}= & \text { RHS of }(2.1)-g(e-\beta-2) \\
& -\left[5\left(\frac{a(\beta+1)}{e}-1\right)\left(\frac{b(\beta+1)}{e}-1\right)\left(\frac{c(\beta+1)}{e}-1\right)\right. \\
& \times\left(\frac{d(\beta+1)}{e}-1\right)-5\left(\frac{d(\beta+1)}{e}-1\right)+5\left(\frac{d}{e}(\beta+1)\right) \\
& \left.\times\left(\frac{d(\beta+1)}{e}-1\right)\left(\frac{d(\beta+1)}{e}-2\right)\left(\frac{d(\beta+1)}{e}-3\right)\right] .
\end{aligned}
$$

Let $A=\frac{a}{e}, B=\frac{b}{e}, C=\frac{c}{e}, D=\frac{d}{e}$ and $\beta=\frac{1}{C}$ and substitute into $\Delta_{20}$. Let $\Delta_{21}=72 C^{4} \Delta_{20}$. From $A(1+\beta) \geq 4, B(1+\beta)>3, C(1+\beta)>2$ and $D(1+$ $\beta)>1$, and $\beta=\frac{1}{D}$, we obtain $A \geq \frac{4 C}{C+1}, B>\frac{3 C}{C+1}, C>1$ and $D>1$. We find that $\Delta_{21}$ is an increasing function with respect to $A, B, C$ and $D$ for $A \geq \frac{4 C}{C+1}, B>\frac{3 C}{C+1}, C>1$ and $D>1$. Evaluated at the minimum $A=\frac{4 C}{C+1}$, $B=\frac{3 C}{C+1}, C=1$ and $D=1, \Delta_{21}>0$, so we can conclude that $\Delta_{20}>0$ for this subcase.

$$
\begin{aligned}
\Delta_{20}= & \frac{1}{72 e^{4}}\left(-180 e a b c-180 e a b d-180 e a c d-180 e b c d+12 a b c d e+1080 d e^{3}\right. \\
& -72 c d e^{4}+63 e^{5} a b c+63 e^{5} a b d+63 e^{5} a c d+63 e^{5} b c d \\
& -198 a b c e^{4}-198 a b d e^{4}-198 a c d e^{4}-198 b c d e^{4} \\
& +1620 a \beta^{2} b e^{2}+2140 a \beta b e^{2}+1620 a \beta^{2} e^{2} c+2140 a \beta e^{2} c \\
& -360 a \beta^{2} e^{2} d+1620 e^{2} b \beta^{2} c+2140 e^{2} b \beta c-360 e^{2} b \beta^{2} d \\
& -360 e^{2} c \beta^{2} d-720 a \beta e^{2} d-720 e^{2} b \beta d+720 d^{3} e-1800 d^{2} e^{2} \\
& -1080 e^{6} \beta-360 \beta^{4} e^{4}-360 \beta^{3} e^{4}+1440 e^{5} \beta^{3} \\
& -2160 e^{6} \beta^{2}+1440 e^{7} \beta+1080 e^{5} \beta^{2}+360 \beta^{2} e^{4} \\
& -720 e^{5} \beta-72 d e^{5}+108 c e^{5}+108 b e^{5}+108 a e^{5}+432 \beta e^{4} \\
& +72 d e^{4}-108 c e^{4}-108 b e^{4}-108 a e^{4}-180 e^{3} a \beta-135 e a b c \beta^{4} \\
& -135 e a b d \beta^{4}-135 e b c d \beta^{4}-135 e a c d \beta^{4}-72 e^{4}-360 e^{2} b d-72 a d e^{4} \\
& -180 e^{3} c \beta^{2}+720 d^{3} \beta^{3} e-180 e^{3} b \beta^{2}-180 e^{3} b \beta \\
& +135 a b c e^{3}+135 a b d e^{3}+135 a c d e^{3}+135 b c d e^{3}-220 e^{3} a b-220 e^{3} a c \\
& -220 e^{3} b c-120 a b c d e^{3}-180 e^{3} c \beta-72 b d e^{4}-360 a e^{2} d-360 e^{5} \\
& -360 e^{8}+360 e^{7}+360 e^{6}-368 a c e^{5}-368 a b e^{5}-368 b c e^{5}+72 b d e^{5} \\
& +72 a d e^{5}+588 a b e^{4}+588 a c e^{4}+588 b c e^{4}+72 c d e^{5}-180 e^{3} a \beta^{2}
\end{aligned}
$$




$$
\begin{aligned}
& +2160 d^{3} \beta^{2} e-1800 d^{2} \beta^{2} e^{2}+2160 d^{3} \beta e-3600 d^{2} \beta e^{2}+1080 d \beta e^{3} \\
& +108 a b c d e^{4}+120 a b c d \beta^{3}-12 a b c d \beta+180 a b c d \beta^{4}+72 a b c d \beta^{5} \\
& -360 c d e^{2}+960 e^{2} b c+960 e^{2} a c+960 e^{2} a b-450 a \beta^{3} b e d-450 a \beta^{3} e c d \\
& -450 e b \beta^{3} c d-450 a \beta^{3} b c e-675 a \beta^{2} b c e-675 a \beta^{2} b e d-540 a \beta b c e \\
& -540 a \beta b e d-675 a \beta^{2} e c d-540 a \beta e c d-675 e b \beta^{2} c d-540 e b \beta c d \\
& \left.-720 e^{2} c \beta d+440 e^{2} a b \beta^{3}+440 e^{2} a c \beta^{3}+440 e^{2} b c \beta^{3} e^{4}\right), \\
& \Delta_{21}=-360-1080 C^{3} e^{2}+960 B C^{5}-135 B C D-810 B C^{2} D-1395 B C^{3} D \\
& -900 B C^{4} D+135 B e^{2} C^{5} D-72 B e^{2} C^{4} D+63 B e^{4} C^{5} D \\
& -198 B e^{3} C^{5} D+72 B e^{3} C^{4} D-900 A C^{4} D+108 A e^{4} B C^{5} D \\
& +63 A e^{4} B C^{4} D-120 A e^{3} B C^{5} D-198 A e^{3} B C^{4} D+135 A e^{2} B C^{4} D \\
& +12 A e B C^{5} D-192 A B C^{4} D-540 A B C^{3} D-555 A B C^{2} D \\
& -270 A B C D-198 A e^{3} B C^{5}+588 A e^{2} B C^{4}-220 A e B C^{4}+420 A B C^{4} \\
& +1465 A B C^{3}+1170 A B C^{2}+305 A B C-180 A B C^{5}+135 A e^{2} B C^{5} \\
& -368 A e^{3} B C^{4}-63 A B D+63 A e^{4} C^{5} D-198 A e^{3} C^{5} D+72 A e^{3} C^{4} D \\
& +135 A e^{2} C^{5} D-72 A e^{2} C^{4} D-180 A C^{5} D-180 B C^{5} D-1395 A C^{3} D \\
& -810 A C^{2} D-135 A C D+720 C^{3} D-360 C^{4} e^{4}+1080 C^{2} e+108 C^{5} e^{2} \\
& -108 C^{5} e-360 C^{4} e+1440 C^{3} e^{3}-720 C^{3} e+360 C^{2}+252 C^{3}-252 C^{4} \\
& +360 C^{4} e^{3}-2160 C^{2} e^{2}+63 A e^{4} B C^{5}-1800 C^{2} D^{2}+2160 C^{2} D^{3} \\
& -360 C^{5} D+2160 C^{3} D^{3}-3600 C^{3} D^{2}+720 C^{4} D^{3}+588 B e^{2} C^{5} \\
& +2140 B C^{4}+1440 B C^{3}+108 A e^{2} C^{4}+72 C^{5} e^{3} D+108 B e^{2} C^{4} \\
& -72 C^{4} e^{2} D-108 B e C^{4}-368 A e^{3} C^{5}-220 B e C^{5}+260 B C^{2} \\
& -368 B e^{3} C^{5}+72 C^{4} e D-108 A e C^{4}+720 C D^{3}+2140 A C^{4}-72 C^{5} e^{2} D \\
& +360 C^{4} D+260 A C^{2}+1440 A C^{3}-1800 C^{4} D^{2}-220 A e C^{5} \\
& +360 C^{4} e^{2}+1440 C e+588 A e^{2} C^{5}+960 A C^{5}-360 C, \\
& \left.\Delta_{21}\right|_{A=\frac{4 C}{C+1}, B=\frac{3 C}{C+1}, C=1, D=1} \\
& =8430-368 e+\frac{1125}{2} e^{4}-2509 e^{3}+\frac{4629}{2} e^{2} \geq 0 \quad \text { for } e \geq 4 \text {. }
\end{aligned}
$$

Case (B3). $\beta=\frac{e}{b}$. Similar to Case (B2). Let $A=\frac{a}{e}, B=\frac{b}{e}, C=\frac{c}{e}, D=\frac{d}{e}$ and $\beta=\frac{1}{B}$ and substitute into $\Delta_{20}$. Let $\Delta_{22}=72 B^{4} \Delta_{20}$. From $\frac{a}{e}(1+\beta) \geq 4$, $\frac{b}{e}(1+\beta)>3, \frac{c}{e}(1+\beta) 2$ and $\frac{d}{e}(1+\beta)>1$. We get $A \geq \frac{4 B}{B+1}, B \geq 2, C \geq$ $\frac{2 B}{B+1}$ and $D \geq 1$. We find that $\Delta_{21}$ is an increasing function with respect to $A, B, C$ and $D$ for $A \geq \frac{4 B}{B+1}, B \geq 2, C \geq \frac{2 B}{B+1} \geq 1$ and $D \geq 1$. Evaluated at the minimum $A=\frac{4 B}{B+1}, B=2, C=1$ and $D=1, \Delta_{22}>0$, so we can 
conclude that $\Delta_{20}>0$ for this subcase.

$$
\begin{aligned}
\Delta_{22}= & -360-720 e B^{3}+108 C e^{2} B^{4}+1440 A B^{3}-108 e A B^{4}+260 C B^{2} \\
& -2160 e^{2} B^{2}-1080 e^{2} B^{3}+360 e^{2} B^{4}+360 e^{3} B^{4}-360 e^{4} B^{4} \\
& +108 B^{5} e^{2}-360 e B^{4}-108 B^{5} e+1440 e^{3} B^{3}+1080 e B^{2}-252 B^{4} \\
& +252 B^{3}+360 B^{2}+260 A B^{2}+108 A e^{2} B^{4}+588 e^{2} B^{5} C \\
& -220 e A B^{5}+720 D^{3} B^{4}-63 A C D+305 A B C-368 B^{5} e^{3} C \\
& +960 A B^{5}+588 e^{2} A B^{5}-198 B^{5} e^{3} C D-1395 C D B^{3} \\
& -810 C D B^{2}+135 B^{5} e^{2} C D-900 C D B^{4}-72 C e^{2} D B^{4} \\
& +72 C e^{3} D B^{4}-198 A e^{3} B^{5} D+63 A e^{4} B^{5} C-180 A B^{5} C \\
& +1170 A C B^{2}+1465 A C B^{3}+420 A C B^{4}+135 A e^{2} B^{5} C \\
& -220 e A C B^{4}+588 e^{2} A C B^{4}-198 A e^{3} B^{5} C-368 A e^{3} C B^{4} \\
& +108 A e^{4} B^{5} C D-198 A e^{3} C D B^{4}+63 A e^{4} C D B^{4} \\
& -120 A e^{3} B^{5} C D+135 A e^{2} C D B^{4}-192 A C D B^{4} \\
& +12 A e B^{5} C D-555 A C D B^{2}-540 A C D B^{3}+1440 B e-220 e B^{5} C \\
& +72 B^{5} e^{3} D+960 B^{5} C-72 B^{5} e^{2} D-135 B C D-135 A B D \\
& +1440 C B^{3}+2140 C B^{4}+2140 A B^{4}-368 A e^{3} B^{5}+63 A e^{4} B^{5} D \\
& -810 A D B^{2}-180 A B^{5} D-1395 A D B^{3}-900 A D B^{4}-72 A e^{2} D B^{4} \\
& +135 A e^{2} B^{5} D+72 A e^{3} D B^{4}-180 B^{5} C D+63 B^{5} e^{4} C D+72 D e B^{4} \\
& -72 D e^{2} B^{4}+360 D B^{4}-1800 D^{2} B^{4}-3600 D^{2} B^{3} \\
& -1800 D^{2} B^{2}+2160 D^{3} B^{2}+2160 D^{3} B^{3}+720 D^{3} B+720 D B^{3} \\
& -360 B^{5} D-108 e C B^{4}-270 A B C D-360 B \\
\Delta_{22} \mid & A=\frac{4 B}{B+1}, B=2, C=1, D=1 \\
& =149634-47136 e+117792 e^{2}-93888 e^{3}+18912 e^{4} \geq 0 \quad \text { for } e \geq 4 .
\end{aligned}
$$

Case (B4). $\beta=\frac{e}{a}$. Similar to Case (B2). Let $A=\frac{a}{e}, B=\frac{b}{e}, C=\frac{c}{e}, D=\frac{d}{e}$ and $\beta=\frac{1}{A}$ and substitute into $\Delta_{20}$. Let $\Delta_{23}=72 A^{4} \Delta_{20}$. From $\frac{a}{e}(1+\beta) \geq 4$, $\frac{b}{e}(1+\beta)>3, \frac{c}{e}(1+\beta)>2$ and $\frac{d}{e}(1+\beta)>1$, we obtain $A \geq 3, B \geq \frac{3 A}{A+1}$, $C \geq \frac{2 A}{A+1}$ and $D \geq 1$. We find that $\Delta_{22}$ is an increasing function with respect to $A, B, C$ and $D$ for $A \geq 3, B \geq \frac{3 A}{A+1}, C \geq \frac{2 A}{A+1}$ and $D \geq 1$, and $e>4$. Evaluated at the minimum $A=3, B=\frac{3 A}{A+1}, C=\frac{2 A}{A+1}$ and $D=1, \Delta_{23}>0$, so we can conclude that $\Delta_{20}>0$ for this subcase.

$$
\begin{aligned}
\Delta_{23}= & -360-2160 e^{2} A^{2}-220 e A^{5} C+108 B e^{2} A^{4}+12 A^{5} e B C D \\
& +360 e^{2} A^{4}+260 C A^{2}+1440 B A^{3}-108 e B A^{4}+260 B A^{2}-368 A^{5} e^{3} C
\end{aligned}
$$




$$
\begin{aligned}
& +2140 B A^{4}+108 C e^{2} A^{4}+2140 C A^{4}+1440 A e+135 B e^{2} C D A^{4} \\
& -192 B C D A^{4}-120 A^{5} e^{3} B C D-555 B C D A^{2}-540 B C D A^{3} \\
& +63 B e^{4} C D A^{4}+108 A^{5} e^{4} B C D-198 B e^{3} C D A^{4}-180 A^{5} B D \\
& -900 B D A^{4}-810 B D A^{2}-72 B e^{2} D A^{4}-1395 B D A^{3}-220 e B C A^{4} \\
& -198 A^{5} e^{3} B C-368 B e^{3} C A^{4}+420 B C A^{4}+135 A^{5} e^{2} B C-180 A^{5} B C \\
& +1465 B C A^{3}+1170 B C A^{2}+588 e^{2} B C A^{4}+63 A^{5} e^{4} B C+63 A^{5} e^{4} B D \\
& +135 A^{5} e^{2} B D-220 e A^{5} B+588 e^{2} A^{5} C-63 B C D-135 A B D \\
& -135 A C D+305 A B C+960 A^{5} B-270 A B C D+588 e^{2} A^{5} B \\
& +63 A^{5} e^{4} C D-198 A^{5} e^{3} C D-180 A^{5} C D-810 C D A^{2}-72 C e^{2} D A^{4} \\
& +135 A^{5} e^{2} C D-900 C D A^{4}-1395 C D A^{3}+72 C e^{3} D A^{4} \\
& -198 A^{5} e^{3} B D+72 B e^{3} D A^{4}-1080 e^{2} A^{3}+1440 e^{3} A^{3} \\
& -720 e A^{3}+360 e^{3} A^{4}-360 e A^{4}-360 e^{4} A^{4}-108 A^{5} e+252 A^{3} \\
& +360 A^{2}+1080 e A^{2}+960 A^{5} C+1440 C A^{3}+720 D A^{3}+720 D^{3} A \\
& +72 A^{5} e^{3} D+360 D A^{4}-1800 D^{2} A^{4}+2160 D^{3} A^{3}-1800 D^{2} A^{2} \\
& + \\
& +2160 D^{3} A^{2}+72 D e A^{4}-72 D e^{2} A^{4}-72 A^{5} e^{2} D \\
& -360 A^{5} D-3600 D^{2} A^{3}+720 D^{3} A^{4}+108 A^{5} e^{2}-108 e C A^{4} \\
& -368 A^{5} e^{3} B-360 A-252 A^{4}, \\
& \left.\Delta_{23}\right|_{A=3, B=} ^{3 A}, C=\frac{2 A}{A+1}, D=1 \\
& =-338553 e+\frac{742851}{4} e^{4}+918072+\frac{3846447}{4} e^{2}-\frac{1647783}{2} e^{3} \\
& +0
\end{aligned}
$$

\section{Acknowledgment}

Research of S.Y. was partially supported by NSF.

\section{References}

[1] A. Durfee, The signature of smoothing of complex surface singularities, Ann. of Math. 232 (1978), 85-98.

[2] E. Ehrhart, Sur un problem de geometrie diophantienne lineaire II, J. Reine Angew. Math. 227 (1967), 25-49.

[3] A. Granville, The lattice points of an n-dimensional tetrahedron, Aequationes Math. 41 (1991), 234-241. 
[4] S. Hong and J. Zhao, Analysis of sharp polynomial upper estimate of number of positive integral points in a five-dimensional tetrahedra, Discrete Math. 308 (2008), 4955-4971.

[5] D.H. Lehmer, The lattice points of an n-dimensional tetrahedron, Duke Math. J. 7 (1940), 341-353.

[6] K.P. Lin, X. Luo, S.S.T. Yau and H. Zuo, On number theoretic conjecture of positive integral points in 5-dimensional tetrahedron and a sharp estimate of Dickman-De Bruijin function, preprint.

[7] K.P. Lin and S.S.T. Yau, Analysis for a sharp polynomial upper estimate of the number of positive integral points in a 4-dimensional tetrahedron, J. Reine Angew. Math. 547 (2002), 191-205.

[8] K.P. Lin and S.S.T. Yau, A sharp estimate of number of integral points in a 5-dimensional tetrahedral, J. Number Theory 93 (2002), 207-234.

[9] K.P. Lin and S.S.T. Yau, Counting the number of integral points in general n-dimensional tetrahedral and Bernoulli polynomials, Can. Math. Bull. 24 (2003), 229-241.

[10] K.P. Lin and S.S.T. Yau, Classification of affine varieties being cones over nonsingular projective varieties: hypersurface case, Comm. Anal. and Geom. 12 (2004), 1201-1219.

[11] X. Luo, S.S.T. Yau and H. Zuo, A sharp estimate of Dickman-De Bruijin function and a sharp estimate of positive integral points in 4-dimensional tetrahedron, 2010.

[12] J. Milnor and P. Orlik, Isolated singularities defined by weighted homogeneous polynomials, Topology 9 (1970), 385-393.

[13] L.J. Mordell, Lattice points in a tetrahedron and generalized Dedekind sums, J. Indian Math. Soc. 15 (1951), 41-46.

[14] M. Merle and B. Teissier, Conditions d'adjonction d'aprěs Du Val, Sĕminaire sur les singularités des surfaces, in 'Center de Math. de l'Ecole Polytechnique, 1976-1977', Lecture Notes in Mathematics, 777, Springer, Berlin, 1980, 229-245

[15] G. Pick, Geometrisches zur Zahlentheorie, Sitzungsber. 19 (1899), 311-319.

[16] O. Saeki, Topological invariance of weights for weighted homogeneous isolated singularities in $\mathbb{C}^{3}$, Proc. Amer. Math. Soc. 103 (1988), 905-909. 
[17] K. Saito, Quasihomogene isolierte Singularititen von Typerflachen, Invent. Math. 14 (1971), 123-142.

[18] X. Wang and S.S.T. Yau, On the GLY Conjecture of upper estimate of positive integral points in real tetrahedra, preprint.

[19] Y.J. Xu and S.S.T. Yau, Classification of topological types of isolated quasihomogeneous two-dimensional hypersurface singularities, Manuscr. Math. 64 (1989), 445-969.

[20] Y.J. Xu and S.S.T. Yau, A sharp estimate of the number of integral points in a tetrahedron, J. Reine Angew. Math. 423 (1992), 199-219.

[21] Y.J. Xu and S.S.T. Yau, Durfee conjecture and coordinate-free characterization of homogeneous singularities, J. Differential Geom. 37 (1993), 375-396.

[22] Y.J. Xu and S.S.T. Yau, Sharp estimate of the number of integral points in a 4-dimensional tetrahedron, J. Reine Angew. Math. 473 (1996), $1-23$.

[23] S.S.T. Yau, Two Theorems in higher dimensional singularities, Math. Ann. 231 (1977), 44-59.

[24] S.S.T. Yau and L. Zhang, An upper estimate of integral points in real simplices with an application to singularity theory, Math. Res. Lett. 13 (2006), 10001-10011.

Massachusetts Institute of Technology

77 Massachusetts Avenue

02139-4307

USA

E-mail address: miss.irene.chen@gmail.com

Department of Information Management

Chang Gung University of Science and Technology

261 WeN HWA 1 ROAD

KWEI-SHAN, TAO-YUAN

TAIWAN

E-mail address: kplin@gw.cgust.edu.tw

Department of Mathematical Science

Tsinghua University,

BEIJING 100084

P. R. China

E-mail address: yau@uic.edu 
Department of Mathematics

Statistics and Computer Science

M/C 249, University of Illinois at Chicago

851 S. Morgan Street

Chicago, IL 60607-7045

USA

E-mail address: hqzuo@uic.edu

ReCeived JAnuARY 7, 2011 